\title{
On the laws of first hitting times of points for one-dimensional symmetric stable Lévy processes
}

\author{
Kouji Yand (1) (2), Yuko YANd (3) $\quad$ and $\quad$ Marc YoR ${ }^{(4)(5)(3)}$
}

November 6, 2018

\begin{abstract}
Several aspects of the laws of first hitting times of points are investigated for one-dimensional symmetric stable Lévy processes. Itô's excursion theory plays a key role in this study.
\end{abstract}

Keywords: Symmetric stable Lévy process, excursion theory, first hitting times.

\section{Introduction}

For one-dimensional Brownian motion, the laws of several random times, such as first hitting times of points and intervals, can be expressed explicitly in terms of elementary functions. Moreover, these laws are infinitely divisible (abbrev. as (ID)), and in fact, self-decomposable (abbrev. as (SD)).

The aim of the present paper is to study various aspects of the laws of first hitting times of points and last exit times for one-dimensional symmetric stable Lévy processes. We shall put some special emphasis on the following objects: (i) the laws of the ratio of two independent gamma variables, which, as is usual, we call beta variables of the second kind; (ii) harmonic transform of Itô's measure of excursions away from the origin. The present study is motivated by a recent work [9] by the authors about penalisations of symmetric stable Lévy paths.

The organisation of the present paper is as follows. In Section 2, we recall several facts concerning beta and gamma variables and their variants. In Section 3, we briefly recall Itô's excursion theory and make some discussions about last exit times. In Section 4. we consider harmonic transforms of symmetric stable Lévy processes, which plays an important role in our study. In Section 5, we discuss the laws of first hitting times of single points and last exit times for symmetric stable Lévy processes. In Section 6, we discuss the laws of those random times for the absolute value of symmetric stable Lévy processes, which includes the reflecting Brownian motion as a special case.

(1) Department of Mathematics, Graduate School of Science, Kobe University, Kobe, Japan. kyano@math.kobe-u.ac.jp

(2) The research of this author is supported by KAKENHI (20740060)

(3) Research Institute for Mathematical Sciences, Kyoto University, Kyoto, Japan.

(4) Laboratoire de Probabilités et Modèles Aléatoires, Université Paris VI, Paris, France.

(5) Institut Universitaire de France 


\section{Preliminaries: several important random variables}

\subsection{Generalized gamma convolutions}

For $a>0$, we write $\mathcal{G}_{a}$ for a gamma variable with parameter $a$ :

$$
P\left(\mathcal{G}_{a} \in \mathrm{d} x\right)=\frac{1}{\Gamma(a)} x^{a-1} \mathrm{e}^{-x} \mathrm{~d} x, \quad x>0 .
$$

As a rather general framework, we recall the class of generalized gamma convolutions (abbrev. as (GGC)), which is an important subclass of (SD); namely,

$$
(\mathrm{GGC}) \subset(\mathrm{SD}) \subset(\mathrm{ID}) \text {. }
$$

A nice reference for details is the monograph [7] by Bondesson. A recent survey can be found in James-Roynette-Yor [26].

A random variable $X$ is said to be of (GGC) type if it is a weak limit of linear combinations of independent gamma variables with positive coefficients.

Theorem 2.1 (See, e.g., [7, Thm.3.1.1]). A random variable $X$ is of (GGC) type if and only if there exist a non-negative constant $a$ and a non-negative measure $U(\mathrm{~d} t)$ on $(0, \infty)$ with

$$
\int_{(0,1]}|\log t| U(\mathrm{~d} t)<\infty \quad \text { and } \quad \int_{(1, \infty)} \frac{1}{t} U(\mathrm{~d} t)<\infty
$$

such that

$$
E\left[\mathrm{e}^{-\lambda X}\right]=\exp \left\{-a \lambda-\int_{(0, \infty)} \log (1+\lambda / t) U(\mathrm{~d} t)\right\}, \quad \lambda>0 .
$$

In what follows we shall call $U(\mathrm{~d} t)$ the Thorin measure associated with $X$.

By simple calculations, it follows that

$$
\begin{aligned}
E\left[\mathrm{e}^{-\lambda X}\right] & =\exp \left\{-a \lambda+\int_{0}^{\infty}\left(\frac{1}{s+\lambda}-\frac{1}{s}\right) U((0, s)) \mathrm{d} s\right\} \\
& =\exp \left\{-a \lambda-\int_{0}^{\infty}\left(1-\mathrm{e}^{-\lambda u}\right) \frac{1}{u}\left(\int_{(0, \infty)} \mathrm{e}^{-u t} U(\mathrm{~d} t)\right) \mathrm{d} u\right\} .
\end{aligned}
$$

In particular, the following holds: The law of $X$ is of (ID) type and its Lévy measure has a density given by $n(u):=\frac{1}{u} \int_{(0, \infty)} \mathrm{e}^{-u t} U(\mathrm{~d} t)$. Since $u n(u)$ is non-increasing, the law of $X$ is of (SD) type.

Theorem 2.2 (see, e.g., [7, Thm.4.1.1 and 4.1.4]). Suppose that $X$ is of (GGC) type and that $a=0$ and $b:=U((0, \infty))<\infty$. Then $X$ may be represented as $X \stackrel{\text { law }}{=} \mathcal{G}_{b} Y$ for some random variable $Y$ independent of $\mathcal{G}_{b}$. The total mass of the Thorin measure is given by

$$
b=\sup \left\{p \geq 0: \lim _{x \rightarrow 0+} \frac{\rho(x)}{x^{p-1}}=0\right\}
$$

where $\rho$ is the density of the law of $X$ with respect to the Lebesgue measure:

$$
\rho(x)=\frac{1}{\Gamma(b)} x^{b-1} E\left[\frac{1}{Y^{b}} \exp \left(-\frac{x}{Y}\right)\right] .
$$


Remark 2.3. For a given $X$, the law of the variable $Y$ which represents $X$ as in Theorem 2.2 is unique; in fact, the gamma distribution is simplifiable (see [11, Sec.1.12]).

Remark 2.4. We do not know how to characterise explicitly the class of possible $Y$ 's which represent variables of (GGC) type as in Theorem 2.2. As a partial converse, Bondesson (see [7, Thm.6.2.1]) has introduced a remarkable class which is closed under multiplication of independent gamma variables.

\subsection{Beta and gamma variables}

We introduce notations and recall several basic facts concerning the beta and gamma variables. See [11, Chap.4] for details. For $a, b>0$, we write $\mathcal{B}_{a, b}$ for a beta variable (of the first kind) with parameters $a, b$ :

$$
P\left(\mathcal{B}_{a, b} \in \mathrm{d} x\right)=\frac{1}{B(a, b)} x^{a-1}(1-x)^{b-1} \mathrm{~d} x, \quad 0<x<1
$$

where $B(a, b)$ is the beta function:

$$
B(a, b)=\frac{\Gamma(a) \Gamma(b)}{\Gamma(a+b)} .
$$

Note that $\mathcal{B}_{a, b} \stackrel{\text { law }}{=} 1-\mathcal{B}_{b, a}$ for $a, b>0$ and that $\mathcal{B}_{a, 1} \stackrel{\text { law }}{=} \mathcal{U}^{\frac{1}{a}}$ for $a>0$ where $\mathcal{U}$ is a uniform variable on $(0,1)$. The following identity in law is well-known: For any $a, b>0$,

$$
\left(\mathcal{G}_{a}, \widehat{\mathcal{G}}_{b}\right) \stackrel{\text { law }}{=}\left(\mathcal{B}_{a, b} \mathcal{G}_{a+b},\left(1-\mathcal{B}_{a, b}\right) \mathcal{G}_{a+b}\right)
$$

or equivalently,

$$
\left(\mathcal{G}_{a}+\widehat{\mathcal{G}_{b}}, \frac{\mathcal{G}_{a}}{\mathcal{G}_{a}+\widehat{\mathcal{G}_{b}}}\right) \stackrel{\text { law }}{=}\left(\mathcal{G}_{a+b}, \mathcal{B}_{a, b}\right)
$$

where on the left hand sides $\mathcal{G}_{a}$ and $\widehat{\mathcal{G}}_{b}$ are independent and on the right hand sides $\mathcal{B}_{a, b}$ and $\mathcal{G}_{a+b}$ are independent. The proof is elementary; it can be seen in [11, (4.2.1)], and so we omit it.

Using the formula (2.11), we obtain another expression of the Thorin measure of a variable of (GGC) type.

Theorem 2.5. Under the same assumption as in Theorem 2.2, the total mass of the Thorin measure is given by

$$
b=\inf \left\{c \geq 0: X \stackrel{\text { law }}{=} \mathcal{G}_{c} Y_{c} \text { for some random variable } Y_{c} \text { independent of } \mathcal{G}_{c}\right\} .
$$

Proof. Let us write $\widetilde{b}$ for the right hand side of (2.13).

By Theorem 2.2, we have $X \stackrel{\text { law }}{=} \mathcal{G}_{b} Y$ for some random variable $Y$ independent of $\mathcal{G}_{b}$. For any $c>b$, we have $\mathcal{G}_{b} \stackrel{\text { law }}{=} \mathcal{G}_{c} \mathcal{B}_{b, c-b}$ where $\mathcal{G}_{c}$ and $\mathcal{B}_{b, c-b}$ are independent, which implies that $c \geq \widetilde{b}$ for any such $c$. Hence we obtain $b \geq \widetilde{b}$. 
Suppose that $b>\widetilde{b}$. Then we may take $c$ with $b>c>\widetilde{b}$ such that $X \stackrel{\text { law }}{=} \mathcal{G}_{c} Z$ for some random variable $Z$ independent of $\mathcal{G}_{c}$. Then we have another expression of the density $\rho(x)$ as

$$
\rho(x)=\frac{1}{\Gamma(c)} x^{c-1} E\left[\frac{1}{Z^{c}} \exp \left(-\frac{x}{Z}\right)\right] .
$$

By the monotone convergence theorem, this implies that

$$
\lim _{x \rightarrow 0+} \frac{\rho(x)}{x^{c-1}}=\frac{1}{\Gamma(c)} E\left[\frac{1}{Z^{c}}\right]>0,
$$

which shows that $c \geq b$ by the formula (2.7). This leads to a contradiction. Therefore we conclude that $b=\widetilde{b}$.

\subsection{Beta variables of the second kind}

Let us consider the ratios of two independent gamma variables, which are sometimes called beta variables of the second kind or beta prime variables. By the identity (2.11), the following is obvious: For any $a, b>0$,

$$
\frac{\mathcal{G}_{a}}{\widehat{\mathcal{G}}_{b}} \stackrel{\text { law }}{=} \frac{\mathcal{B}_{a, b}}{1-\mathcal{B}_{a, b}} .
$$

The law of the ratio $\mathcal{G}_{a} / \widehat{\mathcal{G}}_{b}$ is given as follows: For any $a, b>0$,

$$
P\left(\frac{\mathcal{G}_{a}}{\widehat{\mathcal{G}}_{b}} \in \mathrm{d} x\right)=\frac{1}{B(a, b)} \frac{x^{a-1}}{(1+x)^{a+b}} \mathrm{~d} x, \quad x>0 .
$$

In spite of its simple statement, the following theorem is rather difficult to prove.

Theorem 2.6 (see, e.g., [7, Ex.4.3.1]). For any $a, b>0$, the ratio $\mathcal{G}_{a} / \widehat{\mathcal{G}}_{b}$ is of (GGC) type. Its Thorin measure has total mass a.

For the proof, see [7]. We omit the details.

\section{$2.4 \quad \alpha$-Cauchy variables and Linnik variables}

It is well-known that the standard Cauchy distribution $\frac{1}{\pi} \frac{1}{1+x^{2}} \mathrm{~d} x$ and the bilateral exponential distribution $\frac{1}{2} \mathrm{e}^{-|x|} \mathrm{d} x$ satisfy the following relation:

(R) The characteristic function of one of the two distributions is proportional to the density of the other.

We shall introduce $\alpha$-analogues of these two distributions which satisfy the relation $(\mathbf{R})$.

Let us introduce the $\alpha$-analogue for $\alpha>1$ of the standard Cauchy variable $\mathcal{C}$, which, as just recalled, is given by

$$
P(\mathcal{C} \in \mathrm{d} x)=\frac{1}{\pi} \frac{1}{1+x^{2}} \mathrm{~d} x, \quad x \in \mathbb{R} .
$$


We define the $\alpha$-Cauchy variable $\mathcal{C}_{\alpha}$ as follows:

$$
P\left(\mathcal{C}_{\alpha} \in \mathrm{d} x\right)=\frac{\sin (\pi / \alpha)}{2 \pi / \alpha} \frac{1}{1+|x|^{\alpha}} \mathrm{d} x, \quad x \in \mathbb{R} .
$$

Note that $\mathcal{C}_{2} \stackrel{\text { law }}{=} \mathcal{C}$. By a change of variables, the following is easy to see: For $\alpha>1$, let $\gamma=1 / \alpha \in(0,1)$. Then it holds that

$$
\mathcal{C}_{\alpha}=\epsilon\left(\frac{\mathcal{G}_{\gamma}}{\widehat{\mathcal{G}}_{1-\gamma}}\right)^{\gamma}
$$

where $\epsilon$ is a Bernoulli variable: $P(\epsilon=1)=P(\epsilon=-1)=1 / 2$ independent of $\mathcal{G}_{\gamma}$ and $\widehat{\mathcal{G}}_{1-\gamma}$. In particular,

$$
\frac{\mathcal{G}_{\gamma}}{\widehat{\mathcal{G}}_{1-\gamma}}=\left|\mathcal{C}_{\alpha}\right|^{\alpha}
$$

Note that the law of a standard Cauchy variable $\mathcal{C}_{2}$ is of $(\mathrm{SD})$ type. Moreover, the following theorem is known:

Theorem 2.7 (Bondesson [6]). For $1<\alpha \leq 2$, the law of $\left|\mathcal{C}_{\alpha}\right|$ is of (ID) type.

It is easy to see that

$$
\left|\mathcal{C}_{\alpha}\right| \stackrel{\text { law }}{\longrightarrow} \mathcal{U} \quad \text { as } \alpha \rightarrow \infty
$$

where $\mathcal{U}$ is a uniform variable on $(0,1)$.

Theorem 2.8 (Thorin [42]). For $p>0$, the law of $\mathcal{U}^{-p}$, which is called the Pareto distribution of index $p$, is of (GGC) type.

Remark 2.9. The following problems still remain open:

(i) Is it true that the law of $\mathcal{C}_{\alpha}$ is of (SD) type (or of (ID) type at least)?

(ii) Is it true that the law of $\left|\mathcal{C}_{\alpha}\right|$ is of (SD) type?

(iii) Is it true that the law of $\left|\mathcal{C}_{\alpha}\right|^{-p}$ for $p>0$ is of (SD) type (or of (ID) type at least)?

Remark 2.10. Bourgade-Fujita-Yor ([8]) have proposed a new probabilistic method of computing special values of the Riemann zeta function $\zeta(2 n)$ via the Cauchy variable. Fujita-Y. Yano-Yor [15] have recently generalized their method via the $\alpha$-Cauchy variables and obtained a probabilistic method for computing special values of the complementary sum of the Hurwitz zeta function: $\zeta(2 n, \gamma)+\zeta(2 n, 1-\gamma)$ for $0<\gamma<1$.

Following [12], we introduce the Linnik variable $\Lambda_{\alpha}$ of index $0<\alpha \leq 2$ as follows:

$$
E\left[\mathrm{e}^{i \theta \Lambda_{\alpha}}\right]=\frac{1}{1+|\theta|^{\alpha}}, \quad \theta \in \mathbb{R} .
$$

It is easy to see that

$$
\Lambda_{\alpha} \stackrel{\text { law }}{=} X_{\alpha}(\mathfrak{e})
$$


where $X_{\alpha}=\left(X_{\alpha}(t): t \geq 0\right)$ is the symmetric stable Lévy process of index $\alpha$ starting from 0 such that

$$
P\left[\mathrm{e}^{i \theta X_{\alpha}(t)}\right]=\mathrm{e}^{-t|\theta|^{\alpha}}, \quad \theta \in \mathbb{R}
$$

and $\mathfrak{e}$ is a standard exponential variable independent of $X_{\alpha}$. Hence the laws of Linnik variables are of $(\mathrm{SD})$ type. A Lévy process $\left(\Lambda_{\alpha}(t)\right)$ with $\Lambda_{\alpha}(1) \stackrel{\text { law }}{=} \Lambda_{\alpha}$ is called a Linnik process; its characteristic function is:

$$
E\left[\mathrm{e}^{i \theta \Lambda_{\alpha}(t)}\right]=\frac{1}{\left(1+|\theta|^{\alpha}\right)^{t}}, \quad \theta \in \mathbb{R}
$$

See James [25] for his study of Linnik processes. Note that the law of $\Lambda_{\alpha}$ has a continuous density $L_{\alpha}(x)$, i.e.,

$$
P\left(\Lambda_{\alpha} \in \mathrm{d} x\right)=L_{\alpha}(x) \mathrm{d} x .
$$

Proposition 2.11. Suppose that $1<\alpha<2$. Then the $\alpha$-Cauchy distribution and the Linnik distribution of index $\alpha$ satisfy the relation $(\mathbf{R})$.

Proof. Note that the identities (2.23) and (2.27) show that

$$
\int_{-\infty}^{\infty} \mathrm{e}^{i \theta x} L_{\alpha}(x) \mathrm{d} x=\frac{1}{1+|\theta|^{\alpha}}, \quad \theta \in \mathbb{R} .
$$

By Fourier inversion, we obtain:

$$
L_{\alpha}(x)=\frac{1}{2 \pi} \int_{-\infty}^{\infty} \mathrm{e}^{-i x \theta} \frac{1}{1+|\theta|^{\alpha}} \mathrm{d} \theta, \quad x \in \mathbb{R} .
$$

Hence:

$$
E\left[\mathrm{e}^{i \theta \mathcal{C}_{\alpha}}\right]=\frac{\sin (\pi / \alpha)}{2 \pi / \alpha} L_{\alpha}(\theta), \quad \theta \in \mathbb{R}
$$

Now the proof is complete.

\subsection{Log-gamma processes and their variants}

We recall the classes of log-gamma processes, z-processes and Meixner processes.

It is well-known (see, e.g., [41]) that the law of the logarithm of a gamma variable $\log \mathcal{G}_{a}$ is of (SD) type. Let us introduce a Lévy process $\left(\eta_{a}(t): t \geq 0\right)$ such that

$$
\eta_{a}(1) \stackrel{\operatorname{law}}{=} \log \mathcal{G}_{a} .
$$

Following Carmona-Petit-Yor [10], we call the process $\left(\eta_{a}(t): t \geq 0\right)$ the log-gamma process. Please be careful not to confuse with the convention that log-normal variables stand for exponentials of normal variables. In (2.31), we simply take the logarithm of a gamma variable.

The Lévy characteristics of $\left(\eta_{a}(t): t \geq 0\right)$ are given as follows. 
Theorem 2.12 (see [10] and also [19]). For any $a>0$, the log-gamma process is represented as

$$
\eta_{a}(t) \stackrel{\text { law }}{=} t \Gamma^{\prime}(1)+\sum_{j=0}^{\infty}\left\{\frac{t}{j+1}-\frac{\gamma^{(j)}(t)}{j+a}\right\}
$$

where $\gamma^{(0)}, \gamma^{(1)}, \ldots$ are independent gamma processes. In particular, the Lévy exponent of $\left(\eta_{a}(t): t \geq 0\right)$ defined by

$$
E\left[\mathrm{e}^{i \theta \eta_{a}(t)}\right]=\left(\frac{\Gamma(a+i \theta)}{\Gamma(a)}\right)^{t}=\mathrm{e}^{t \phi_{a}(\theta)}
$$

admits the representation

$$
\begin{aligned}
\phi_{a}(\theta) & =\log \frac{\Gamma(a+i \theta)}{\Gamma(a)} \\
& =i \theta \psi(a)+\int_{-\infty}^{0}\left(\mathrm{e}^{i \theta u}-1-i \theta u\right) \frac{\mathrm{e}^{-a|u|}}{|u|\left(1-\mathrm{e}^{-|u|}\right)} \mathrm{d} u
\end{aligned}
$$

where $\psi(z)=\Gamma^{\prime}(z) / \Gamma(z)$ is called the digamma function.

Let $\left(\eta_{a}(t): t \geq 0\right)$ and $\left(\widehat{\eta}_{b}(t): t \geq 0\right)$ be independent log-gamma processes. Then the difference $\left(\eta_{a}(t)-\widehat{\eta}_{b}(t): t \geq 0\right)$ is called a generalized $z$-process (see [18]). In particular, we have

$$
\eta_{a}(1)-\widehat{\eta}_{b}(1) \stackrel{\operatorname{law}}{=} \log \frac{\mathcal{G}_{a}}{\widehat{\mathcal{G}}_{b}}
$$

and this law is called a $z$-distribution. Its characteristic function is given by

$$
E\left[\exp \left\{i \theta \log \frac{\mathcal{G}_{a}}{\widehat{\mathcal{G}}_{b}}\right\}\right]=\frac{B(a+i \theta, b-i \theta)}{B(a, b)}
$$

and the law itself is given by

$$
P\left(\log \frac{\mathcal{G}_{a}}{\widehat{\mathcal{G}}_{b}} \in \mathrm{d} x\right)=\frac{1}{B(a, b)} \frac{\mathrm{e}^{a x}}{\left(1+\mathrm{e}^{x}\right)^{a+b}} \mathrm{~d} x .
$$

\subsection{Symmetric $z$-processes}

We now consider a particular case of symmetric $z$-processes, i.e.,

$$
\sigma_{a}(t)=\frac{1}{\pi}\left\{\eta_{a}(t)-\widehat{\eta}_{a}(t)\right\}, \quad t \geq 0
$$

We introduce a subordinator given by

$$
\Sigma_{a}(t)=\frac{2}{\pi^{2}} \sum_{j=0}^{\infty} \frac{\gamma^{(j)}(t)}{(j+a)^{2}}
$$


where $\gamma^{(0)}, \gamma^{(1)}, \ldots$ are independent gamma processes. The Lévy measure of $\left(\Sigma_{a}(t): t \geq 0\right)$ may be obtained from the following:

$$
\begin{aligned}
E\left[\mathrm{e}^{-\lambda \Sigma_{a}(t)}\right] & =\prod_{j=0}^{\infty} E\left[\exp \left\{-\lambda \frac{2 \gamma^{(j)}(t)}{\pi^{2}(j+a)^{2}}\right\}\right] \\
& =\prod_{j=0}^{\infty} \exp \left\{-t \int_{0}^{\infty}\left(1-\exp \left(-\frac{2 \lambda u}{\pi^{2}(j+a)^{2}}\right)\right) \mathrm{e}^{-u} \frac{\mathrm{d} u}{u}\right\} \\
& =\prod_{j=0}^{\infty} \exp \left\{-t \int_{0}^{\infty}\left(1-\mathrm{e}^{-\lambda u}\right) \exp \left(-\frac{\pi^{2}(j+a)^{2}}{2} u\right) \frac{\mathrm{d} u}{u}\right\} \\
& =\exp \left\{-t \int_{0}^{\infty}\left(1-\mathrm{e}^{-\lambda u}\right) n(u) \mathrm{d} u\right\}
\end{aligned}
$$

where

$$
n(u)=\frac{1}{u} \sum_{j=0}^{\infty} \exp \left(-\frac{\pi^{2}(j+a)^{2}}{2} u\right)=\frac{1}{u} \int_{(0, \infty)} \mathrm{e}^{-u t} U(\mathrm{~d} t)
$$

with

$$
U=\sum_{j=0}^{\infty} \delta_{\pi^{2}(j+a)^{2} / 2}
$$

Hence we conclude that the law of $\Sigma_{a}(t)$ for fixed $t$ is of (GGC) type.

The following theorem, due to Barndorff-Nielsen-Kent-Sørensen [1], connects the two Lévy processes $\Sigma_{a}$ and $\sigma_{a}$ :

Theorem 2.13 ([1]; see, e.g., [10]). The process $\left(\sigma_{a}(t): t \geq 0\right)$ may be obtained as the subordination of a Brownian motion $(\widehat{B}(u): u \geq 0)$ with respect to the subordinator $\left(\Sigma_{a}(t): t \geq 0\right)$ :

$$
\sigma_{a}(t) \stackrel{\text { law }}{=} \widehat{B}\left(\Sigma_{a}(t)\right), \quad t \geq 0
$$

Proof. By (2.32), the process $\left(\sigma_{a}(t): t \geq 0\right)$ is represented as

$$
\sigma_{a}(t)=\frac{1}{\pi} \sum_{j=0}^{\infty} \frac{\widehat{\gamma}^{(j)}(t)-\gamma^{(j)}(t)}{j+a}
$$

where $\gamma^{(0)}, \gamma^{(1)}, \ldots, \widehat{\gamma}^{(0)}, \widehat{\gamma}^{(1)}, \ldots$ are independent gamma processes. Note that

$$
\widehat{\gamma}^{(j)}(t)-\gamma^{(j)}(t) \stackrel{\text { law }}{=} \Lambda_{2}(t) \stackrel{\text { law }}{=} \sqrt{2} \widehat{B}(\gamma(t))
$$

where $\left(\Lambda_{2}(t): t \geq 0\right)$ is a Linnik process, i.e., a Lévy process such that $\Lambda_{2}(1) \stackrel{\text { law }}{=} \Lambda_{2}$. Now 
we obtain

$$
\begin{aligned}
\widehat{B}\left(\Sigma_{a}(t)\right) & \stackrel{\text { law }}{=} \frac{\sqrt{2}}{\pi} \sum_{j=0}^{\infty} \frac{\widehat{B}_{j}\left(\gamma^{(j)}(t)\right)}{j+a} \\
& \stackrel{\text { law }}{=} \frac{1}{\pi} \sum_{j=0}^{\infty} \frac{\widehat{\gamma}^{(j)}(t)-\gamma^{(j)}(t)}{j+a} \\
& \stackrel{\text { law }}{=} \sigma_{a}(t)
\end{aligned}
$$

where on the right hand side of (2.50) the $\widehat{B}_{j}$ 's are independent of $\gamma^{(j)}$ 's. The proof is completed.

The characteristic function of $\sigma_{a}(t)$ is given by

$$
E\left[\mathrm{e}^{i \theta \sigma_{a}(t)}\right]=\left(E\left[\exp \left\{i \frac{\theta}{\pi} \log \frac{\mathcal{G}_{a}}{\widehat{\mathcal{G}}_{a}}\right\}\right]\right)^{t}=\mathrm{e}^{-t \Phi_{a}(\theta)}
$$

where

$$
\begin{aligned}
\Phi_{a}(\theta) & =\int_{-\infty}^{\infty}\left(1-\mathrm{e}^{i \theta u}\right) \frac{\mathrm{e}^{-a \pi|u|}}{|u|\left(1-\mathrm{e}^{-\pi|u|}\right)} \mathrm{d} u \\
& =2 \int_{0}^{\infty}(1-\cos \theta u) \frac{\mathrm{e}^{-a \pi u}}{u\left(1-\mathrm{e}^{-\pi u}\right)} \mathrm{d} u .
\end{aligned}
$$

For $t=1$, the law of $\sigma_{a}(1)$ is given by

$$
P\left(\sigma_{a}(1) \in \mathrm{d} x\right)=P\left(\frac{1}{\pi} \log \frac{\mathcal{G}_{a}}{\widehat{\mathcal{G}}_{a}} \in \mathrm{d} x\right)=\frac{\pi}{B(a, a)} \frac{\mathrm{e}^{a \pi x}}{\left(1+\mathrm{e}^{\pi x}\right)^{2 a}} \mathrm{~d} x
$$

Example 2.14. When $a=1 / 2$, let us denote

$$
C_{t}:=\Sigma_{\frac{1}{2}}(t), \quad \mathbb{C}_{t}:=\widehat{B}\left(C_{t}\right)=\sigma_{\frac{1}{2}}(t) .
$$

The law of $\mathbb{C}_{1}$ is called the hyperbolic cosine distribution:

$$
E\left[\mathrm{e}^{i \theta \mathbb{C}_{1}}\right]=E\left[\mathrm{e}^{-\frac{1}{2} \theta^{2} C_{1}}\right]=\frac{1}{\cosh \theta}, \quad P\left(\mathbb{C}_{1} \in \mathrm{d} x\right)=\frac{1}{\cosh \pi x} \mathrm{~d} x .
$$

Consequently, $\mathbb{C}_{1}$ and $\pi \mathbb{C}_{1}$ satisfy the relation (R).

Example 2.15. When $a=1$, let us denote

$$
S_{t}:=\Sigma_{1}(t), \quad \mathbb{S}_{t}:=\widehat{B}\left(S_{t}\right)=\sigma_{1}(t) .
$$

The law of $\mathbb{S}_{1}$ is called the logistic distribution:

$$
E\left[\mathrm{e}^{i \theta \mathbb{S}_{1}}\right]=E\left[\mathrm{e}^{-\frac{1}{2} \theta^{2} S_{1}}\right]=\frac{\theta}{\sinh \theta}, \quad P\left(\mathbb{S}_{1} \in \mathrm{d} x\right)=\frac{\pi}{(\cosh \pi x)^{2}} \mathrm{~d} x .
$$

Consequently, $\mathbb{S}_{1}$ and $\pi \mathbb{C}_{2}$ satisfy the relation $(\mathbf{R})$. 
Let us introduce a subordinator $\left(T_{t}\right)$ and then $\left(\mathbb{T}_{t}\right)$ such that

$$
\mathbb{T}_{t}=\widehat{B}\left(T_{t}\right)
$$

and that

$$
E\left[\mathrm{e}^{i \theta \mathbb{T}_{t}}\right]=E\left[\mathrm{e}^{-\frac{1}{2} \theta^{2} T_{t}}\right]=\left(\frac{\tanh \theta}{\theta}\right)^{t}
$$

It is well-known that the law of $T_{1}$ is of (ID) type and hence that such processes exist. Now it is obvious that

$$
C_{t} \stackrel{\text { law }}{=} T_{t}+S_{t}, \quad \mathbb{C}_{t} \stackrel{\text { law }}{=} \mathbb{T}_{t}+\mathbb{S}_{t}
$$

where $\left(T_{t}\right)$ and $\left(S_{t}\right)$ are independent and so are $\left(\mathbb{T}_{t}\right)$ and $\left(\mathbb{S}_{t}\right)$.

For further study of these processes $C_{t}, S_{t}$ and $T_{t}$, see Pitman-Yor [32]. By taking Laplace inversion, the density of the law of $T_{1}$ can be obtained in terms of theta function; see Knight [28, Cor.2.1] for details.

\subsection{Meixner processes}

Let $\beta \in(-\pi, \pi)$ and let $\left(\mathcal{M}_{\beta}(t): t \geq 0\right)$ be a Lévy process such that

$$
\mathcal{M}_{\beta}(1) \stackrel{\text { law }}{=} \frac{1}{2 \pi} \log \frac{\mathcal{G}_{a}}{\widehat{\mathcal{G}}_{1-a}} \quad \text { where } \beta=(2 a-1) \pi
$$

The law of $\mathcal{M}_{\beta}(t)$ for fixed $t$ is called a Meixner distribution because of its close relation to Meixner-Pollaczek polynomials (See [38, [39], [40] and [17]). The characteristic function of $\mathcal{M}_{\beta}(t)$ is given by

$$
E\left[\mathrm{e}^{i \theta \mathcal{M}_{\beta}(t)}\right]=\left(\frac{\cos \frac{\beta}{2}}{\cosh \frac{\theta-i \beta}{2}}\right)^{t}=\mathrm{e}^{t \xi_{\beta}(\theta)}
$$

where

$$
\xi_{\beta}(\theta)=\frac{i \theta}{2 \pi}\left\{\psi\left(\frac{\pi+\beta}{2 \pi}\right)-\psi\left(\frac{\pi-\beta}{2 \pi}\right)\right\}+\int_{-\infty}^{\infty}\left(\mathrm{e}^{i \theta u}-1-i \theta u\right) \frac{\mathrm{e}^{\beta u}}{u \sinh (\pi u)} \mathrm{d} u .
$$

The law of $\mathcal{M}_{\beta}(t)$ itself is given by

$$
\begin{aligned}
P\left(\mathcal{M}_{\beta}(t) \in \mathrm{d} x\right) & =\frac{\left(2 \cos \frac{\beta}{2}\right)^{t}}{2 \pi \Gamma(t)} \mathrm{e}^{\beta x}\left|\Gamma\left(\frac{t}{2}+i x\right)\right|^{2} \mathrm{~d} x \\
& =\frac{\left(2 \cos \frac{\beta}{2}\right)^{t}}{2 \pi \Gamma(t)} \mathrm{e}^{\beta x} \Gamma(t / 2)^{2} \mathrm{e}^{-\Phi_{t / 2}(x)} \mathrm{d} x \\
& =\frac{\left(2 \cos \frac{\beta}{2}\right)^{t} B(t / 2, t / 2)}{2 \pi} \mathrm{e}^{\beta x-\Phi_{t / 2}(x)} \mathrm{d} x
\end{aligned}
$$


where $\Phi_{a}(x)$ has been defined in (2.55).

We simply write $\mathcal{M}_{\beta}$ for $\mathcal{M}_{\beta}(1)$. Remark that this Meixner distribution is identical to that of the $\log$ of an $\alpha$-Cauchy variable:

$$
\mathcal{M}_{\beta} \stackrel{\text { law }}{=} \frac{\alpha}{2 \pi} \log \left|\mathcal{C}_{\alpha}\right| \quad \text { where } \beta=\left(\frac{2}{\alpha}-1\right) \pi .
$$

Remark also that the law of $\mathcal{M}_{\beta}$ is symmetric if and only if $\beta=0$ (or $a=1 / 2$ ). Then the corresponding Meixner distribution is identical to the hyperbolic cosine distribution, up to the factor $1 / 2$; precisely:

$$
\mathcal{M}_{0} \stackrel{\text { law }}{=} \frac{1}{\pi} \log |\mathcal{C}| \stackrel{\text { law }}{=} \frac{1}{2 \pi} \log \frac{\mathcal{G}_{1 / 2}}{\widehat{\mathcal{G}}_{1 / 2}} \stackrel{\text { law }}{=} \frac{1}{2} \mathbb{C}_{1}
$$

\section{$2.8 \alpha$-Rayleigh distributions}

For an exponential variable $\mathfrak{e}$, the random variable

$$
\mathcal{R}=\sqrt{2 \mathfrak{e}}
$$

is sometimes called a Rayleigh variable. We shall introduce an $\alpha$-analogue of the Rayleigh variable.

Let $0<\alpha \leq 2$ and let $t>0$. By Fourier inversion, we obtain from (2.25) that

$$
P\left(X_{\alpha}(t) \in \mathrm{d} x\right)=p_{t}^{(\alpha)}(x) \mathrm{d} x
$$

where

$$
p_{t}^{(\alpha)}(x)=\frac{1}{2 \pi} \int_{-\infty}^{\infty} \mathrm{e}^{-i x \xi} \mathrm{e}^{-t|\xi|^{\alpha}} \mathrm{d} \xi=\frac{1}{\pi} \int_{0}^{\infty} \cos (x \xi) \mathrm{e}^{-t \xi^{\alpha}} \mathrm{d} \xi
$$

Note that

$$
p_{1}^{(\alpha)}(0)=\frac{\Gamma(1 / \alpha)}{\alpha \pi}
$$

Lemma 2.16. Let $0<\alpha \leq 2$. Then there exists a non-negative random variable $\mathcal{R}_{\alpha}$ such that

$$
P\left(\mathcal{R}_{\alpha}>x\right)=\frac{p_{1}^{(\alpha)}(x)}{p_{1}^{(\alpha)}(0)}, \quad x>0 .
$$

In particular, $\mathcal{R}_{2}=\sqrt{2} \mathcal{R}=2 \sqrt{\mathfrak{e}}$.

We call $\mathcal{R}_{\alpha}$ an $\alpha$-Rayleigh variable and its law the $\alpha$-Rayleigh distribution.

For the proof of Lemma 2.16, we introduce some notations. For $0<\alpha<1$, we denote by $\mathcal{T}_{\alpha}$ the unilateral $\alpha$-stable distribution:

$$
E\left[\mathrm{e}^{-\lambda \mathcal{T}_{\alpha}}\right]=\mathrm{e}^{-\lambda^{\alpha}}, \quad \lambda \geq 0
$$

We denote by $\mathcal{T}_{\alpha}^{\prime}$ the $h$-size biased variable of $\mathcal{T}_{\alpha}$ with respect to $h(x)=x^{-1 / 2}$ :

$$
E\left[f\left(\mathcal{T}_{\alpha}^{\prime}\right)\right]=\frac{E\left[\left(\mathcal{T}_{\alpha}\right)^{-1 / 2} f\left(\mathcal{T}_{\alpha}\right)\right]}{E\left[\left(\mathcal{T}_{\alpha}\right)^{-1 / 2}\right]}
$$

for any non-negative Borel function $f$. The following lemma proves Lemma 2.16. 
Lemma 2.17. Let $0<\alpha<2$. Then the variable $\mathcal{R}_{\alpha}$ is given by

$$
\mathcal{R}_{\alpha}=2 \sqrt{\mathfrak{e} \mathcal{T}_{\alpha / 2}^{\prime}}
$$

where the variables $\mathfrak{e}$ and $\mathcal{T}_{\alpha / 2}^{\prime}$ are independent.

Proof of Lemma 2.17. Since we have

$$
X_{\alpha}(1) \stackrel{\text { law }}{=} \sqrt{2} \widehat{B}\left(\mathcal{T}_{\frac{\alpha}{2}}\right)
$$

we obtain the following expression:

$$
p_{1}^{(\alpha)}(x)=E\left[\frac{1}{2 \sqrt{\pi \mathcal{T}_{\frac{\alpha}{2}}}} \exp \left\{-\frac{x^{2}}{4 \mathcal{T}_{\frac{\alpha}{2}}}\right\}\right] .
$$

Hence we obtain

$$
\frac{p_{1}^{(\alpha)}(x)}{p_{1}^{(\alpha)}(0)}=E\left[\exp \left\{-\frac{x^{2}}{4 \mathcal{T}_{\frac{\alpha}{2}}^{\prime}}\right\}\right]
$$

Using an independent exponential variable $\mathfrak{e}$, we have

$$
\frac{p_{1}^{(\alpha)}(x)}{p_{1}^{(\alpha)}(0)}=E\left[\mathfrak{e}>\frac{x^{2}}{4 \mathcal{T}_{\frac{\alpha}{2}}^{\prime}}\right]=E\left[2 \sqrt{\mathfrak{e} \mathcal{T}_{\frac{\alpha}{2}}^{\prime}}>x\right] .
$$

Now the proof is complete.

\section{Discussions from excursion theoretic viewpoint}

Let us recall Itô's excursion theory ([23] and [30]). See also the standard textbooks [22] and [35], as well as [33].

\subsection{Itô's measure of excursions away from the origin}

We simply write $\mathbb{D}$ for the space $\mathbb{D}([0, \infty) ; \mathbb{R})$ of càdlàg paths equipped with Skorokhod topology. Let $X=(X(t): t \geq 0)$ be a strong Markov process with paths in $\mathbb{D}$ starting from 0. Suppose that the origin is regular, recurrent and an instantaneous state. Then it is well-known (see [4, Thm.V.3.13]) that there exists a local time at the origin, which we denote by $L=(L(t): t \geq 0)$, subject to the normalization:

$$
E\left[\int_{0}^{\infty} \mathrm{e}^{-t} \mathrm{~d} L(t)\right]=1
$$

This is a choice made in this section; but later, we may make another choice, which will be indicated as $L^{(\alpha)}(t), L(t)$ being always subject to (3.1). The local time process 
$L=(L(t): t \geq 0)$ is continuous and non-decreasing almost surely. Thus its rightcontinuous inverse process

$$
\tau(l)=\inf \{t>0: L(t)>l\}
$$

is strictly-increasing. By the strong Markov property of $X$, we see that $\tau(l)$ is a subordinator. We define a random set $D$ to be the set of discontinuities of $\tau$ :

$$
D=\{l>0: \tau(l)-\tau(l-)>0\} .
$$

It is obvious that $D$ is a countable set. Now we define a point function $\boldsymbol{p}$ on $D$ which takes values in $\mathbb{D}$ as follows: For $l \in D$,

$$
\boldsymbol{p}(l)(t)= \begin{cases}X(t+\tau(l-)) & \text { if } 0 \leq t<\tau(l)-\tau(l-), \\ 0 & \text { otherwise. }\end{cases}
$$

We call $\boldsymbol{p}=(\boldsymbol{p}(l): l \in D)$ the excursion point process. Then the fundamental theorem of Itô's excursion theory is stated as follows.

Theorem 3.1 (Itô [23]; see also Meyer [30]). The excursion point process $\boldsymbol{p}$ is a Poisson point process, i.e.:

(i) $\boldsymbol{p}$ is $\sigma$-discrete almost surely, i.e., for almost every sample path, there exists a sequence $\left\{U_{n}\right\}$ of disjoint measurable subsets of $\mathbb{D}$ such that $\mathbb{D}=\cup_{n} U_{n}$ and $\left\{l \in D: \boldsymbol{p}(l) \in U_{n}\right\}$ is a finite set for all $n$;

(ii) $\boldsymbol{p}$ is renewal, i.e., $\boldsymbol{p}(\cdot \wedge s)$ and $\boldsymbol{p}(\cdot+s)$ are independent for each $s>0$.

For a measurable subset $U$ of $\mathbb{D}$, we define a point process $\boldsymbol{p}_{U}: D_{U} \rightarrow \mathbb{D}$ as

$$
D_{U}=\{l \in D: \boldsymbol{p}(l) \in U\} \quad \text { and } \quad \boldsymbol{p}_{U}=\left.\boldsymbol{p}\right|_{D_{U}} .
$$

We call $\boldsymbol{p}_{U}=\left(\boldsymbol{p}_{U}(l): l \in D_{U}\right)$ the restriction of $\boldsymbol{p}$ on $U$. The measure on $\mathbb{D}$ defined by

$$
\boldsymbol{n}(U)=E\left[\sharp\left((0,1] \cap D_{U}\right)\right]
$$

is called Itô's measure of excursions.

Corollary 3.2 (Itô [23]). The following statements hold:

(i) Let $\left\{U_{n}\right\}$ be a sequence of disjoint measurable subsets of $\mathbb{D}$. Then the point processes $\left\{\boldsymbol{p}_{U_{n}}\right\}$ are independent;

(ii) Let $U$ be a measurable subset of $\mathbb{D}$ such that $\boldsymbol{n}(U)<\infty$. Then $(0, l] \cap D_{U}$ is a finite set for all $l>0$ a.s. Set

$$
D_{U}=\left\{0<\kappa_{1}<\kappa_{2}<\cdots\right\}, \quad \boldsymbol{p}_{U}\left(\kappa_{n}\right)=u_{n}, n=1,2, \ldots
$$

Then:

(ii-a) $\left\{\kappa_{n}-\kappa_{n-1}, u_{n}: n=1,2, \ldots\right\}$ are independent where $\kappa_{0}=0$;

(ii-b) For each $n, \kappa_{n}-\kappa_{n-1}$ is exponentially distributed with mean $1 / \boldsymbol{n}(U)$, i.e., $P\left(\kappa_{n}-\right.$ $\left.\kappa_{n-1}>l\right)=\mathrm{e}^{-\boldsymbol{n} \boldsymbol{n}(U)}$ for $l>0$; 
(ii-c) For each $n, P\left(u_{n} \in \cdot\right)=\boldsymbol{n}(\cdot \cap U) / \boldsymbol{n}(U)$;

(iii) Let $F(l, u)$ be a non-negative measurable functional on $(0, \infty) \times \mathbb{D}$. Then

$$
E\left[\exp \left\{-\sum_{l \in D} F(l, \boldsymbol{p}(l))\right\}\right]=\exp \left\{-\int\left(1-\mathrm{e}^{-F(l, u)}\right) \mathrm{d} l \otimes \boldsymbol{n}(\mathrm{d} u)\right\}
$$

(iv) Let $F(t, u)$ be a non-negative measurable functional on $(0, \infty) \times \mathbb{D}$. Then

$$
E\left[\sum_{l \in D} F(\tau(l-), \boldsymbol{p}(l))\right]=\int E[F(\tau(l), u)] \mathrm{d} l \otimes \boldsymbol{n}(\mathrm{d} u) .
$$

The proofs of Theorem 3.1 and Corollary 3.2 are also found in [22] and [35].

For $u \in \mathbb{D}$, define

$$
\zeta(u)=\sup \{t \geq 0: u(t) \neq 0\} .
$$

For each excursion path $\boldsymbol{p}(l), l \in D, \zeta(\boldsymbol{p}(l))$ is finite and called the lifetime of the path $\boldsymbol{p}(l)$. For a measurable subset $U$ of $\mathbb{D}$, we set

$$
\tau_{U}(l)=\sum_{k \in(0, l] \cap D_{U}} \zeta\left(\boldsymbol{p}_{U}(k)\right) .
$$

Note that $\tau_{\mathbb{D}}(l)=\tau(l), l \geq 0$. By Corollary 3.2 (iii), we see that the process $\left(\tau_{U}(l): l \geq 0\right)$ is a subordinator with Laplace transform $E\left[\mathrm{e}^{-\lambda \tau_{U}(l)}\right]=\mathrm{e}^{-l \psi_{U}(\lambda)}$ given by

$$
\psi_{U}(\lambda)=\boldsymbol{n}\left[1-\mathrm{e}^{-\lambda \zeta} ; U\right], \quad \lambda>0
$$

Since $\psi(\lambda):=\psi_{\mathbb{D}}(\lambda)<\infty$, we have $\boldsymbol{n}(\zeta>t)<\infty$ for all $t>0$; in particular, we see that the measure $\boldsymbol{n}$ is $\sigma$-finite.

\subsection{Decomposition of first hitting time before and after last exit time}

We denote the first hitting time of a closed set $F$ for $X$ by

$$
T_{F}(X)=\inf \{t>0: X(t) \in F\} .
$$

In particular, if $F=\{a\}$, the closed set consisting of a single point $a \in \mathbb{R}, T_{F}(X)$ is nothing else but the first hitting time of point $a \in \mathbb{R}$ for $X$ :

$$
T_{\{a\}}(X)=\inf \{t>0: X(t)=a\} .
$$

The hitting time $T_{\{a\}}(X)$ may be decomposed at the last exit time from 0 ;

$$
T_{\{a\}}(X)=G_{\{a\}}(X)+\Xi_{\{a\}}(X)
$$

where $G_{\{a\}}(X)$ is the last exit time from 0 before $T_{\{a\}}(X)$, and where $\Xi_{\{a\}}(X)$ is the remainder of time after $G_{\{a\}}(X)$, i.e.,

$$
G_{\{a\}}(X)=\sup \left\{t \leq T_{\{a\}}(X): X(t)=0\right\}, \quad \Xi_{\{a\}}(X)=T_{\{a\}}(X)-G_{\{a\}}(X) .
$$

The joint law of the random times $G_{\{a\}}(X)$ and $\Xi_{\{a\}}(X)$ is characterised by the following proposition: 
Proposition 3.3. Let $a \neq 0$. Then the random times $G_{\{a\}}(X)$ and $\Xi_{\{a\}}(X)$ are independent. Moreover, the law of $G_{\{a\}}(X)$ is of (ID) type. The Laplace transforms of $G_{\{a\}}(X)$ and $\Xi_{\{a\}}(X)$ are given as

$$
E\left[\mathrm{e}^{-\lambda G_{\{a\}}(X)}\right]=\left\{1+\frac{\boldsymbol{n}\left[1-\mathrm{e}^{-\lambda \zeta} ; T_{\{a\}}>\zeta\right]}{\boldsymbol{n}\left(T_{\{a\}}<\zeta\right)}\right\}^{-1}
$$

and

$$
E\left[\mathrm{e}^{-\lambda \Xi_{\{a\}}(X)}\right]=\frac{\boldsymbol{n}\left[\mathrm{e}^{-\lambda T_{\{a\}}} ; T_{\{a\}}<\zeta\right]}{\boldsymbol{n}\left(T_{\{a\}}<\zeta\right)} .
$$

Consequently, the Laplace transform of $T_{\{a\}}(X)$ is given as

$$
E\left[\mathrm{e}^{-\lambda T_{\{a\}}(X)}\right]=\frac{\boldsymbol{n}\left[\mathrm{e}^{-\lambda T_{\{a\}}} ; T_{\{a\}}<\zeta\right]}{\boldsymbol{n}\left[1-\left(\mathrm{e}^{-\lambda \zeta} \cdot 1_{\left\{T_{\{a\}}>\zeta\right\}}\right)\right]} .
$$

Proof. Set

$$
U_{a}=\left\{u \in \mathbb{D}: T_{\{a\}}(u)<\infty\right\} .
$$

By Corollary 3.2 (i), we see that $\boldsymbol{p}_{U_{a}^{c}}$ and $\boldsymbol{p}_{U_{a}}$ are independent. We remark that $\boldsymbol{n}\left(U_{a}\right)<$ $\infty$; in fact, if we supposed otherwise, then there would exist a sequence $\left\{t_{n}\right\}$ such that $t_{n} \rightarrow 0$ decreasingly and that $X\left(t_{n}\right)=a$, which contradicts $X(0+)=X(0)=0$. Set

$$
\kappa_{a}=\inf \left\{l>0: \boldsymbol{p}(l) \in U_{a}\right\} .
$$

Then, by Corollary 3.2 (ii), we see that $\kappa_{a}$ and $\boldsymbol{p}\left(\kappa_{a}\right)$ are independent. Since $\kappa_{a}=\inf D_{U_{a}}$ and $\boldsymbol{p}\left(\kappa_{a}\right)=\boldsymbol{p}_{U_{a}}\left(\kappa_{a}\right)$, they are measurable with respect to the $\sigma$-field generated by $\boldsymbol{p}_{U_{a}}$. Hence we see that $\left\{\boldsymbol{p}_{U_{a}^{c}}, \kappa_{a}, \boldsymbol{p}\left(\kappa_{a}\right)\right\}$ are independent. Note that

$$
G_{\{a\}}(X)=\tau_{U_{a}^{c}}\left(\kappa_{a}\right) \quad \text { and } \quad \Xi_{\{a\}}(X)=T_{\{a\}}\left(\boldsymbol{p}\left(\kappa_{a}\right)\right) .
$$

Thus we conclude that $G_{\{a\}}(X)$ and $\Xi_{\{a\}}(X)$ are independent. Moreover, we see that the law of $G_{\{a\}}(X)$ is of (ID) type; in fact, $\tau_{U_{a}^{c}}$ is a subordinator with Laplace exponent $\psi_{U_{a}^{c}}(\lambda)$ and $\kappa_{a}$ is an exponential variable with mean $1 / \boldsymbol{n}\left(U_{a}\right)$ independent of $\tau_{U_{a}^{c}}$. The law of $\Xi_{\{a\}}(X)$ is given by

$$
P\left(\Xi_{\{a\}}(X) \in \cdot\right)=\frac{\boldsymbol{n}\left(u \in \mathbb{D}: T_{\{a\}}(u) \in \cdot\right)}{\boldsymbol{n}\left(U_{a}\right)} .
$$

Now the proof is completed.

\subsection{Excursion durations}

Let us consider the excursion straddling $t$. For a general study in the setup of linear diffusions, see [37]. 
We define the last exit time from 0 before $t$ and the first hitting time of point 0 after $t$ as follows:

$$
G_{t}(X)=\sup \{s \leq t: X(s)=0\} \quad \text { and } \quad D_{t}(X)=\inf \{s>t: X(s)=0\} .
$$

We define

$$
\Xi_{t}(X)=t-G_{t}(X) \quad \text { and } \quad \Delta_{t}(X)=D_{t}(X)-G_{t}(X) .
$$

We recall (see (3.1) $)$ that $L=(L(t): t \geq 0)$ denotes the local time at 0 of $X$ and $\tau=(\tau(l): l \geq 0)$ its right-continuous inverse. Then we have

$$
G_{t}(X)=\tau(L(t)-), \quad D_{t}(X)=\tau(L(t))
$$

and

$$
\Xi_{t}(X)=t-\tau(L(t)-), \quad \Delta_{t}(X)=\tau(L(t))-\tau(L(t)-) .
$$

If the local time process $L$ has the self-similarity property with index $\gamma$ :

$$
\left(\frac{L(c t)}{c^{\gamma}}: t \geq 0\right) \stackrel{\text { law }}{=}(L(t): t \geq 0), \quad c>0
$$

then we have

$$
\left\{\left(\frac{L(c t)}{c^{\gamma}}: t \geq 0\right),\left(\frac{\tau\left(c^{\gamma} l\right)}{c}: l \geq 0\right)\right\} \stackrel{\text { law }}{=}\{(L(t): t \geq 0),(\tau(l): l \geq 0)\}
$$

for any $c>0$; in particular, $\tau$ is a stable subordinator of index $\gamma$. Hence the index $\gamma$ must be in $(0,1)$.

We now state two explicit results, the proofs of which are postponed after commenting about these results.

Theorem 3.4. Suppose that the local time process has the self-similarity property of index $0<\gamma<1$. Then

$$
\left(\Xi_{1}(X), \Delta_{1}(X)\right) \stackrel{\text { law }}{=}\left(\mathcal{B}_{1-\gamma, \gamma}, \frac{\mathcal{B}_{1-\gamma, \gamma}}{\mathcal{U}^{\frac{1}{\gamma}}}\right)
$$

where $\mathcal{B}_{1-\gamma, \gamma}$ is a beta variable of index $(1-\gamma, \gamma)$ and $\mathcal{U}$ is an independent uniform variable on $(0,1)$.

The following is a special case of Winkel [45, Cor.1]:

Theorem 3.5 ([45]). Suppose that the local time process has the self-similarity property of index $0<\gamma<1$. Let $\mathfrak{e}$ be an independent exponential time. Then

$$
\left(G_{\mathfrak{e}}(X), \Xi_{\mathfrak{e}}(X), \Delta_{\mathfrak{e}}(X)\right) \stackrel{\text { law }}{=}\left(\mathcal{G}_{\gamma}, \widehat{\mathcal{G}}_{1-\gamma}, \frac{\widehat{\mathcal{G}}_{1-\gamma}}{\mathcal{U}^{\frac{1}{\gamma}}}\right)
$$

where $\mathcal{G}_{\gamma}$ and $\widehat{\mathcal{G}}_{1-\gamma}$, respectively, are independent gamma variables of indeces $\gamma$ and $1-\gamma$, respectively, and $\mathcal{U}$ is an independent uniform variable. 
Generalizing a self-decomposability result of Bondesson (see [7, Ex.5.6.3]), BertoinFujita-Roynette-Yor [3, Thm.1.1] and Roynette-Vallois-Yor [36, Thm.5] have recently proved the following:

Theorem 3.6 ([3] and [36]). For any $\gamma \in(0,1)$, the laws

$$
\frac{\mathcal{G}_{1-\gamma}}{\mathcal{U}^{\frac{1}{\gamma}}} \quad \text { and } \quad\left(\frac{1}{\mathcal{U}^{\frac{1}{\gamma}}}-1\right) \mathcal{G}_{1-\gamma}
$$

are both of (GGC) type with their Thorin measures having total mass $1-\gamma$. Here $\mathcal{G}_{1-\gamma}$ is a gamma variable of index $1-\gamma$ and $\mathcal{U}$ is an independent uniform variable.

Example 3.7. For a symmetric stable Lévy process of index $\alpha$, it is well-known (see Kesten [27] and Bretagnolle [9]) that the origin is regular for itself if and only if $1<\alpha \leq 2$. Let $X_{\alpha}=\left(X_{\alpha}(t): t \geq 0\right)$ be the symmetric stable Lévy process of index $1<\alpha \leq 2$. Then its local time process is given as

$$
L(t)=\lim _{\varepsilon \rightarrow 0+} \frac{C}{2 \varepsilon} \int_{0}^{t} 1_{\left\{\left|X_{\alpha}(s)\right|<\varepsilon\right\}} \mathrm{d} s
$$

for some constant $C$. Since $X$ satisfies the self-similarity property with index $1 / \alpha$, so does $L$ with index $1-1 / \alpha$, and hence Theorems 3.4 and 3.5 hold with $\gamma=1-1 / \alpha$.

Example 3.8. For a Bessel process of dimension $d$, it is well-known that the origin is regular for itself if and only if $0<d<2$. Let $X=(X(t): t \geq 0)$ be a reflecting Bessel process starting from 0 of dimension $d=2-2 \alpha, 0<d<2$ (or $0<\alpha<1$ ) which is scaled so that it has natural scale and speed measure $m(0, x)=x^{\frac{1}{\alpha}-1}$. Then its local time process is given as

$$
L(t)=\lim _{\varepsilon \rightarrow 0+} \frac{C}{m(0, \varepsilon)} \int_{0}^{t} 1_{\{|X(s)|<\varepsilon\}} \mathrm{d} s
$$

for some constant $C$. Since $X$ satisfies the self-similarity property with index $\alpha$, so does $L$ with the same index $\alpha$, and hence Theorems 3.4 and 3.5 hold with $\gamma=\alpha$. For the relations among several choices in the literature, see [13].

Example 3.9. Let $\alpha>0$ and $0<\beta<\min \{1,1 / \alpha\}$ and consider the process $X=$ $X_{m^{(\alpha)}, j^{(\beta)}, 0,0}$ given in [47, Ex.2.4.(b)]. Then $X$ satisfies the self-similarity property with index $\alpha$, but this property seems to have nothing to do with the local time $L$. Since its inverse local time process $\tau=\eta_{m^{(\alpha)}, j^{(\beta)}, 0,0}$ satisfies the self-similarity property with index $1 /(\alpha \beta)$, so does $L$ with index $\alpha \beta$, and hence Theorems 3.4 and 3.5 hold with $\gamma=\alpha \beta$.

Remark 3.10. The identities in law $\Xi_{1}\left(X_{\alpha}\right) \stackrel{\text { law }}{=} \mathcal{B}_{\gamma, 1-\gamma}$ and $D_{1}\left(X_{\alpha}\right)-1 \stackrel{\text { law }}{=} \mathcal{G}_{\gamma} / \widehat{\mathcal{G}}_{1-\gamma}$ are found in Feller [14, XIV.3] as the long-time limit laws of similar random variables derived from random walks.

Let us prove Theorems 3.4 and 3.5 for completeness of this paper. 
Proof of Theorem 3.4. Since $\tau\left(c^{\gamma} l\right) \stackrel{\text { law }}{=} c \tau(l)$ for $c, l>0$, we have $\psi(c \lambda)=c^{\gamma} \psi(\lambda)$. Hence we obtain

$$
\boldsymbol{n}(\zeta \in \mathrm{d} t)=C \frac{\mathrm{d} t}{t^{\gamma+1}}
$$

for some constant $C$. For $t>0$, the excursion straddling time $t$ is $\boldsymbol{p}(L(t))$. Hence we have

$$
G_{t}=\tau(L(t)-), \quad \Xi_{t}=t-\tau(L(t)-), \quad \Delta_{t}=\zeta(\boldsymbol{p}(L(t))) .
$$

Let $p, q, r$ be positive constants. Then

$$
\begin{aligned}
E\left[\int_{0}^{\infty} \mathrm{e}^{-p t-q \Xi_{t}-r \Delta_{t}} \mathrm{~d} t\right] & =E\left[\sum_{l \in D} \int_{\tau(l-)}^{\tau(l)} \mathrm{e}^{-p t-q \Xi_{t}-r \Delta_{t}} \mathrm{~d} t\right] \\
& =E\left[\sum_{l \in D} \mathrm{e}^{-p \tau(l-)-r \zeta(\boldsymbol{p}(l))} \int_{0}^{\zeta(\boldsymbol{p}(l))} \mathrm{e}^{-p t-q t} \mathrm{~d} t\right] \\
& =\int_{0}^{\infty} E\left[\mathrm{e}^{-p \tau(l)}\right] \mathrm{d} l \int_{\mathbb{D}} \boldsymbol{n}(\mathrm{d} u) \mathrm{e}^{-r \zeta(u)} \int_{0}^{\zeta(u)} \mathrm{e}^{-p t-q t} \mathrm{~d} t \\
& =\int_{0}^{\infty} \mathrm{e}^{-l \psi(p)} \mathrm{d} l \int_{0}^{\infty} C \frac{\mathrm{d} s}{s^{\gamma+1}} \mathrm{e}^{-r s} \int_{0}^{s} \mathrm{e}^{-p t-q t} \mathrm{~d} t \\
& =\frac{C}{\psi(p)} \int_{0}^{\infty} \mathrm{d} t \mathrm{e}^{-p t-q t} \int_{t}^{\infty} \frac{\mathrm{d} s}{s^{\gamma+1}} \mathrm{e}^{-r s} .
\end{aligned}
$$

Note that

$$
\frac{1}{\psi(p)}=\frac{1}{\psi(1) p^{\gamma}}=\frac{1}{\psi(1) \Gamma(\gamma)} \int_{0}^{\infty} t^{\gamma-1} \mathrm{e}^{-p t} \mathrm{~d} t
$$

Hence we have

$$
\begin{aligned}
\text { (3.41) } & =\frac{C}{\psi(1) \Gamma(\gamma)} \int_{0}^{\infty} \mathrm{d} t \mathrm{e}^{-p t} \int_{0}^{t} \mathrm{~d} v(t-v)^{\gamma-1} \mathrm{e}^{-q v} \int_{v}^{\infty} \frac{\mathrm{d} s}{s^{\gamma+1}} \mathrm{e}^{-r s} \\
& =\frac{C}{\psi(1) \Gamma(\gamma)} \int_{0}^{\infty} \mathrm{d} t \mathrm{e}^{-p t} \int_{0}^{1} \mathrm{~d} v v^{-\gamma}(1-v)^{\gamma-1} \mathrm{e}^{-q v t} \int_{1}^{\infty} \frac{\mathrm{d} s}{s^{\gamma+1}} \mathrm{e}^{-r s v t} \\
& =C^{\prime} \int_{0}^{\infty} \mathrm{d} t \mathrm{e}^{-p t} E\left[\exp \left\{-q \mathcal{B}_{1-\gamma, \gamma} t-r \frac{\mathcal{B}_{1-\gamma, \gamma}}{\mathcal{U}^{\frac{1}{\gamma}}} t\right\}\right] \\
& =C^{\prime} E\left[\frac{1}{p+q \mathcal{B}_{1-\gamma, \gamma}+r \mathcal{B}_{1-\gamma, \gamma} \mathcal{U}^{-1 / \gamma}}\right]
\end{aligned}
$$

for some constant $C^{\prime}$.

On the other hand, by the self-similarity property $\left([3.29)\right.$, we have $\left(\Xi_{t}, \Delta_{t}\right) \stackrel{\text { law }}{=}\left(t \Xi_{1}, t \Delta_{1}\right)$ for fixed $t>0$, and hence we have

$$
E\left[\int_{0}^{\infty} \mathrm{e}^{-p t-q \Xi_{t}-r \Delta_{t}} \mathrm{~d} t\right]=E\left[\frac{1}{p+q \Xi_{1}+r \Delta_{1}}\right] .
$$

Letting $q, r \rightarrow 0+$ and comparing (3.46) and (3.47), we have $C^{\prime}=1$. Therefore we obtain the desired identity in law (3.30) by the uniqueness property of Stieltjes transform. 
Proof of Theorem 3.5. Note that $\left(G_{\mathfrak{e}}, \Xi_{\mathfrak{e}}, \Delta_{\mathfrak{e}}\right) \stackrel{\text { law }}{=}\left(\mathfrak{e} G_{1}, \mathfrak{e} \Xi_{1}, \mathfrak{e} \Delta_{1}\right)$ by the self-similarity property (3.29) . We also note that $\left(\mathfrak{e}\left(1-\mathcal{B}_{1-\gamma, \gamma}\right), \mathfrak{e} \mathcal{B}_{1-\gamma, \gamma}\right) \stackrel{\text { law }}{=}\left(\mathcal{G}_{\gamma}, \widehat{\mathcal{G}}_{1-\gamma}\right)$ by the identity in law (2.11). Therefore we obtain the desired identity in law (3.31) as an immediate consequence of Theorem 3.4 .

\section{Harmonic transforms of symmetric stable Lévy processes}

We keep the notation $X_{\alpha}=\left(X_{\alpha}(t): t \geq 0\right)$ for the symmetric stable Lévy process of index $\alpha$ such that

$$
P\left[\mathrm{e}^{i \theta X_{\alpha}(t)}\right]=\mathrm{e}^{-t|\theta|^{\alpha}}, \quad \theta \in \mathbb{R}
$$

Note that, with (4.1), we have $X_{2}(t) \stackrel{\text { law }}{=} \sqrt{2} B(t)$. We have

$$
P\left(X_{\alpha}(t) \in \mathrm{d} x\right)=p_{t}^{(\alpha)}(x) \mathrm{d} x
$$

where

$$
p_{t}^{(\alpha)}(x)=\frac{1}{2 \pi} \int_{-\infty}^{\infty} \mathrm{e}^{-i x \xi} \mathrm{e}^{-t|\xi|^{\alpha}} \mathrm{d} \xi=\frac{1}{\pi} \int_{0}^{\infty} \cos (x \xi) \mathrm{e}^{-t \xi^{\alpha}} \mathrm{d} \xi .
$$

We suppose that $1<\alpha \leq 2$. Then the Laplace transform

$$
u_{q}^{(\alpha)}(x)=\int_{0}^{\infty} \mathrm{e}^{-q t} p_{t}^{(\alpha)}(x) \mathrm{d} t=\frac{1}{\pi} \int_{0}^{\infty} \frac{\cos (x \xi)}{q+\xi^{\alpha}} \mathrm{d} \xi
$$

is finite. Define

$$
h_{q}^{(\alpha)}(x)=u_{q}^{(\alpha)}(0)-u_{q}^{(\alpha)}(x), \quad q>0, x \in \mathbb{R}
$$

and

$$
h^{(\alpha)}(x)=\lim _{q \rightarrow 0+} h_{q}^{(\alpha)}(x)=\lim _{q \rightarrow 0+}\left\{u_{q}^{(\alpha)}(0)-u_{q}^{(\alpha)}(x)\right\}, \quad x \in \mathbb{R} .
$$

Lemma 4.1 (See also [29, Sec.4.2]). Suppose that $1<\alpha \leq 2$. Then the following assertions hold:

(i) $u_{q}^{(\alpha)}(0)=u_{1}^{(\alpha)}(0) q^{\frac{1}{\alpha}-1}$ for any $q>0$ where $u_{1}^{(\alpha)}(0)=\frac{1}{\alpha \pi} \Gamma\left(1-\frac{1}{\alpha}\right) \Gamma\left(\frac{1}{\alpha}\right)$;

(ii) $h^{(\alpha)}(x)=h^{(\alpha)}(1)|x|^{\alpha-1}$ for any $x \in \mathbb{R}$ where $h^{(\alpha)}(1)=\left\{2 \Gamma(\alpha) \sin \frac{(\alpha-1) \pi}{2}\right\}^{-1}$;

(iii) $\lim _{q \rightarrow 0+} \frac{u_{q}^{(\alpha)}(x)}{u_{q}^{(\alpha)}(0)}=1$ for any $x \in \mathbb{R}$.

Proof. The assertion (i) is obvious by definition. It is also obvious that

$$
h^{(\alpha)}(x)=\frac{1}{\pi} \int_{0}^{\infty} \frac{1-\cos (x \xi)}{\xi^{\alpha}} \mathrm{d} \xi=h^{(\alpha)}(1)|x|^{\alpha-1}, \quad x \in \mathbb{R} .
$$


For the computation:

$$
h^{(\alpha)}(1)=\frac{1}{\pi} \int_{0}^{\infty} \frac{1-\cos \xi}{\xi^{\alpha}} \mathrm{d} \xi=\left\{2 \Gamma(\alpha) \sin \frac{(\alpha-1) \pi}{2}\right\}^{-1}
$$

see Proposition 7.1 in the Appendix. Hence we obtain (ii). We obtain the assertion (iii) noting that

$$
\frac{u_{q}^{(\alpha)}(x)}{u_{q}^{(\alpha)}(0)}=1-\frac{h_{q}^{(\alpha)}(x)}{u_{q}^{(\alpha)}(0)} \stackrel{q \rightarrow 0+}{\longrightarrow} 1
$$

Let $\left(L^{(\alpha)}(t): t \geq 0\right)$ be the unique local time process such that

$$
L^{(\alpha)}(t)=\lim _{\varepsilon \rightarrow 0+} \frac{1}{2 \varepsilon} \int_{0}^{t} 1_{\left\{\left|X_{\alpha}(s)\right|<\varepsilon\right\}} \mathrm{d} s \quad \text { a.s. }
$$

Then it is well-known (see [2, Lemma V.1.3]) that

$$
E\left[\int_{0}^{\infty} \mathrm{e}^{-t} \mathrm{~d} L^{(\alpha)}(t)\right]=u_{1}^{(\alpha)}(0)
$$

Let $\boldsymbol{n}^{(\alpha)}$ denote Itô's measure for the process $X_{\alpha}$ corresponding to this normalisation of the local time $\left(L^{(\alpha)}(t): t \geq 0\right)$. Remark that

$$
L^{(\alpha)}(t)=u_{1}^{(\alpha)}(0) L(t) \quad(t \geq 0), \quad \boldsymbol{n}^{(\alpha)}=\frac{1}{u_{1}^{(\alpha)}(0)} \boldsymbol{n}
$$

where $(L(t): t \geq 0)$ and $\boldsymbol{n}$, respectively, are as defined by (3.1) and (3.6), respectively.

Theorem 4.2 ([49] and [48]). Suppose that $1<\alpha \leq 2$. Then

$$
\boldsymbol{n}^{(\alpha)}\left[h^{(\alpha)}(X(t)) ; \zeta>t\right]=1, \quad t>0 .
$$

Consequently, there exists a unique probability measure $P^{h^{(\alpha)}}$ on $\mathbb{D}$ such that

$$
E^{h^{(\alpha)}}\left[Z_{t}\right]=\boldsymbol{n}^{(\alpha)}\left[Z_{t} h^{(\alpha)}(X(t)) ; \zeta>t\right]
$$

for any $t>0$ and for any non-negative or bounded $\mathcal{F}_{t}$-measurable functional $Z_{t}$.

The proof of Theorem 4.2 can be found in [49, Thm.4.7], so we omit it. See [48, Thm.1.2] for the proof of Theorem 4.2 for a fairly general class of one-dimensional symmetric Lévy processes. Several aspects of the law of local time process will be discussed in Hayashi-K. Yano [20]. 
Example 4.3. In the case where $\alpha=2$, we have $X_{2}(t)=\sqrt{2} B(t)$, and we have the following formulae:

$$
\begin{aligned}
& p_{t}^{(2)}(x)=\frac{1}{2 \sqrt{\pi t}} \mathrm{e}^{-\frac{x^{2}}{4 t}}, \quad t>0, x \in \mathbb{R}, \\
& u_{q}^{(2)}(x)=\frac{1}{2 \sqrt{q}} \mathrm{e}^{-\sqrt{q}|x|}, \quad q>0, x \in \mathbb{R}, \\
& h^{(2)}(x)=\frac{1}{2}|x|, \quad x \in \mathbb{R} .
\end{aligned}
$$

The process $\left(\frac{1}{\sqrt{2}} X(t): t \geq 0\right)$ under $P^{h^{(2)}}$ is nothing else but the symmetrised 3-dimensional Bessel process starting from the origin.

Theorem 4.4 ([48]). Let $q>0$. Then the following assertions are valid:

(i) Suppose that $1<\alpha \leq 2$. Then it holds that

$$
\lim _{x \rightarrow 0} \frac{h_{q}^{(\alpha)}(x)}{h^{(\alpha)}(x)}=1 ;
$$

(iia) Suppose that $1<\alpha<2$. Let $a \neq 0$. Then it holds that

$$
\lim _{x \rightarrow 0} \frac{u_{q}^{(\alpha)}(a-x)-u_{q}^{(\alpha)}(a)}{h^{(\alpha)}(x)}=0 ;
$$

(iib) Suppose that $\alpha=2$. Let $a \neq 0$. Then it holds that

$$
\lim _{x \rightarrow \pm 0} \frac{u_{q}^{(2)}(a-x)-u_{q}^{(2)}(a)}{h^{(2)}(x)}= \pm \mathrm{e}^{-\sqrt{q}|a|} .
$$

The proof of the claim (i) of Theorem 4.4 can be found in [48, Lem.4.4], so we omit it. The proof of the claim (iib) of Theorem 4.4 is immediate from the formulae (4.16) and (4.17), so we omit it, too. The proof of the claim (iia) of Theorem 4.4 is immediate from the following estimate:

Lemma $4.5([48])$. Suppose that $1<\alpha<2$. Let $a, x \in \mathbb{R}$ with $0<2|x|<|a|$. Then there exists a constant $C_{q}^{(\alpha)}$ such that

$$
\left|u_{q}^{(\alpha)}(a-x)-u_{q}^{(\alpha)}(a)\right| \leq \frac{C_{q}^{(\alpha)}}{|a|} .
$$

The proof of Lemma 4.5 can be found in [48, Lem.6.2, (i)] in a rather general setting, but we give it for convenience of the reader.

Proof of Lemma 4.5. Integrating by parts, we have

$$
\begin{aligned}
u_{q}^{(\alpha)}(a-x)-u_{q}^{(\alpha)}(a) & =\frac{1}{\pi} \int_{0}^{\infty} \frac{\cos a \xi-\cos (a-x) \xi}{q+\xi^{\alpha}} \mathrm{d} \xi \\
& =\frac{1}{\pi} \int_{0}^{\infty}\{\varphi(a \xi)-\varphi((a-x) \xi)\} \frac{\alpha \xi^{\alpha} \mathrm{d} \xi}{\left(q+\xi^{\alpha}\right)^{2}}
\end{aligned}
$$


where

$$
\varphi(x)=\frac{\sin x}{x} \quad(x \neq 0), \quad \varphi(0)=1 .
$$

Since $\varphi^{\prime}(x)=\frac{\cos x}{x}-\frac{\sin x}{x^{2}}(x \neq 0)$, we have

$$
|\varphi(a \xi)-\varphi((a-x) \xi)| \leq\left|\int_{(a-x) \xi}^{a \xi}\right| \varphi^{\prime}(y)|\mathrm{d} y| \leq\left|\int_{(a-x) \xi}^{a \xi} \frac{2}{|y|} \mathrm{d} y\right| .
$$

We change variables: $y=u \xi$, then we have

$$
|\varphi(a \xi)-\varphi((a-x) \xi)| \leq\left|\int_{a-x}^{a} \frac{2}{|u|} \mathrm{d} u\right| \leq \frac{4|x|}{|a|} .
$$

Thus we have proved the estimate (4.21).

Let us prove the claim (iia) of Theorem 4.4.

Proof of the claim (iia) of Theorem 4.4. Without loss of generality, we may suppose that $0<2|x|<|a|$. Using the estimate (4.21), we obtain

$$
\left|\frac{u_{q}^{(\alpha)}(a-x)-u_{q}^{(\alpha)}(a)}{h^{(\alpha)}(x)}\right| \leq \frac{C_{q}^{(\alpha)}}{|a|} \cdot \frac{|x|^{2-\alpha}}{h^{(\alpha)}(1)},
$$

which tends to zero since $\alpha<2$. Now the proof is complete.

\section{$5 \quad$ First hitting time of a single point for $X_{\alpha}$}

\subsection{The case of one-dimensional Brownian motion}

Let $B=(B(t): t \geq 0)$ denote the one-dimensional Brownian motion starting from 0 . We consider the first hitting time of $a \in \mathbb{R}$ for $B$ :

$$
T_{\{a\}}(B)=\inf \{t>0: B(t)=a\} .
$$

It is well-known (see, e.g., [35, Prop.II.3.7]) that the law of the hitting time is of (SD) type where its Laplace transform is given as follows:

$$
E\left[\mathrm{e}^{i \theta \widehat{B}\left(T_{\{a\}}(B)\right)}\right]=E\left[\mathrm{e}^{-\frac{1}{2} \theta^{2} T_{\{a\}}(B)}\right]=\mathrm{e}^{-|a \theta|}, \quad \theta \in \mathbb{R}
$$

where $\widehat{B}=(\widehat{B}(t): t \geq 0)$ stands for an independent copy of $B$. The identity (5.2) can be expressed as

$$
\widehat{B}\left(T_{\{a\}}(B)\right) \stackrel{\text { law }}{=}|a| \mathcal{C}, \quad T_{\{a\}}(B) \stackrel{\text { law }}{=} 2 a^{2} \mathcal{T}_{\frac{1}{2}} .
$$

Let $a>0$. Let us consider the random times $G_{\{a\}}(B)$ and $\Xi_{\{a\}}(B)$. The following path decomposition is due to Williams (see [43] and [44]; see also Prop.VII.4.8 and Thm.VII.4.9 of [35]): 
Theorem 5.1 ([43] and [44]). The process $\left(B(t): 0 \leq t \leq T_{\{a\}}(B)\right)$ is identical in law to the process $\left(Y(t): 0 \leq t \leq T^{\prime}\right)$ defined as follows:

$$
Y(t)= \begin{cases}B_{1}(t) & \text { for } 0 \leq t<T_{\{M\}}\left(B_{1}\right) \\ B_{2}(T-t) & \text { for } T_{\{M\}}\left(B_{1}\right) \leq t<T \\ R(T+t) & \text { for } T \leq t \leq T^{\prime}\end{cases}
$$

where $M, B_{1}, B_{2}$ and $R$ are independent, $M$ is a uniform variable on $(0, a), B_{1}$ and $B_{2}$ are both identical in law to $B, R$ is a 3-dimensional Bessel process starting at 0 , and $T$ and $T^{\prime}$ are random times defined as follows:

$$
T=T_{\{M\}}\left(B_{1}\right)+T_{\{M\}}\left(B_{2}\right), \quad T^{\prime}=T+T_{\{a\}}(R) .
$$

From this path decomposition, we may compute the Laplace transform of $G_{\{a\}}(B)$ as follows:

$$
E\left[\mathrm{e}^{-q G_{\{a\}}(B)}\right]=\int_{0}^{a} \frac{\mathrm{d} m}{a}\left(E\left[\mathrm{e}^{-q T_{\{m\}}(B)}\right]\right)^{2}=\frac{1-\mathrm{e}^{-2 \sqrt{2 q} a}}{2 \sqrt{2 q} a}, \quad q>0 .
$$

We may also compute the Laplace transform of $\Xi_{\{a\}}(B)$ as follows:

$$
E\left[\mathrm{e}^{-q \Xi_{\{a\}}(B)}\right]=E\left[\mathrm{e}^{-q T_{\{a\}}(R)}\right]=\frac{\sqrt{2 q} a}{\sinh (\sqrt{2 q} a)}, \quad q>0 .
$$

In other words, we have

$$
\Xi_{\{a\}}(B) \stackrel{\text { law }}{=} T_{\{a\}}(R) \stackrel{\text { law }}{=} a^{2} S_{1} .
$$

Remark 5.2. The laws of first hitting times are known to be of (SD) type also for Bessel processes with drift (see Pitman-Yor [31]) and of (ID) type for one-dimensional diffusion processes (see Yamazato [46] and references therein).

\subsection{The law of $T_{\{a\}}\left(X_{\alpha}\right)$}

Let us consider the first hitting time of point $a \in \mathbb{R}$ for $X_{\alpha}$ of index $1<\alpha \leq 2$ :

$$
T_{\{a\}}\left(X_{\alpha}\right)=\inf \left\{t>0: X_{\alpha}(t)=a\right\} .
$$

It is well-known (see, e.g., [2, Cor.II.5.18]) that

$$
E\left[\mathrm{e}^{-q T_{\{a\}}\left(X_{\alpha}\right)}\right]=\frac{u_{q}^{(\alpha)}(a)}{u_{q}^{(\alpha)}(0)}, \quad q>0 .
$$

Let $\widehat{X}_{\alpha}=\left(\widehat{X}_{\alpha}(t): t \geq 0\right)$ be an independent copy of $X_{\alpha}$. The following is a generalization of the formulae (5.2) and (5.3) . 
Theorem 5.3. Suppose that $1<\alpha \leq 2$. Let $a \in \mathbb{R}$. Then

$$
E\left[\mathrm{e}^{i \theta \widehat{X}_{\alpha}\left(T_{\{a\}}\left(X_{\alpha}\right)\right)}\right]=E\left[\mathrm{e}^{-|\theta|^{\alpha} T_{\{a\}}\left(X_{\alpha}\right)}\right]=\frac{\sin (\pi / \alpha)}{2 \pi / \alpha} L_{\alpha}(a \theta)
$$

and

$$
\widehat{X}_{\alpha}\left(T_{\{a\}}\left(X_{\alpha}\right)\right) \stackrel{\text { law }}{=}|a| \mathcal{C}_{\alpha}, \quad T_{\{a\}}\left(X_{\alpha}\right) \stackrel{\text { law }}{=} \frac{|a|^{\alpha}}{\left(\mathcal{R}_{\alpha}\right)^{\alpha} \mathcal{B}_{1-\gamma, \gamma}}
$$

where $\gamma=1 / \alpha$.

We can recover (5.3) if we take $\alpha=2$, noting that

$$
T_{\{a\}}(B) \stackrel{\text { law }}{=} T_{\{\sqrt{2} a\}}\left(X_{2}\right) \stackrel{\text { law }}{=} \frac{2 a^{2}}{\left(\mathcal{R}_{2}\right)^{2} \mathcal{B}_{1 / 2,1 / 2}} \stackrel{\text { law }}{=} \frac{a^{2}}{2 \mathfrak{e} \mathcal{B}_{1 / 2,1 / 2}} \stackrel{\text { law }}{=} \frac{a^{2}}{2 \mathcal{G}_{1 / 2}} \stackrel{\text { law }}{=} 2 a^{2} \mathcal{T}_{1 / 2} .
$$

Proof of Theorem 5.3. If we take $q=|\theta|^{\alpha}$, then

$$
u_{q}^{(\alpha)}(x)=\frac{1}{\pi} \int_{0}^{\infty} \frac{\cos (x \xi)}{|\theta|^{\alpha}+|\xi|^{\alpha}} \mathrm{d} \xi=\frac{|\theta|^{1-\alpha}}{\pi} \int_{0}^{\infty} \frac{\cos (\theta x \xi)}{1+|\xi|^{\alpha}} \mathrm{d} \xi, \quad x \in \mathbb{R} .
$$

Hence, by the formula (5.10), we obtain

$$
E\left[\mathrm{e}^{-|\theta|^{\alpha} T_{\{a\}}\left(X_{\alpha}\right)}\right]=E\left[\cos \left(\theta|a| \mathcal{C}_{\alpha}\right)\right]=E\left[\mathrm{e}^{i \theta|a| \mathcal{C}_{\alpha}}\right] .
$$

This shows (5.11) and the first identity of (5.12).

To prove the second identity of (15.12), it suffices to prove the claim when $a=1$; in fact, by the self-similarity property, we have

$$
T_{\{a\}}\left(X_{\alpha}\right) \stackrel{\text { law }}{=}|a|^{\alpha} T_{\{1\}}\left(X_{\alpha}\right) .
$$

Note that

$$
\int_{0}^{\infty} \mathrm{e}^{-q t} P\left(T_{\{1\}}\left(X_{\alpha}\right)<t\right) \mathrm{d} t=\frac{1}{q} E\left[\mathrm{e}^{-q T_{\{1\}}\left(X_{\alpha}\right)}\right]=\frac{u_{q}^{(\alpha)}(1)}{q u_{q}^{(\alpha)}(0)} .
$$

Since $u_{q}^{(\alpha)}(0)=u_{1}^{(\alpha)}(0) q^{\gamma-1}$ where $\gamma=\frac{1}{\alpha}$, we have

$$
\frac{1}{q u_{q}^{(\alpha)}(0)}=\frac{1}{u_{1}^{(\alpha)}(0)} q^{-\gamma}=\frac{1}{u_{1}^{(\alpha)}(0) \Gamma(\gamma)} \int_{0}^{\infty} y^{\gamma-1} \mathrm{e}^{-q y} \mathrm{~d} y
$$

Hence, by Laplace inversion, we obtain

$$
P\left(T_{\{1\}}\left(X_{\alpha}\right)<t\right)=\frac{1}{u_{1}^{(\alpha)}(0) \Gamma(\gamma)} \int_{0}^{t}(t-s)^{\gamma-1} p_{s}^{(\alpha)}(1) \mathrm{d} s .
$$

By the scaling property

$$
p_{s}^{(\alpha)}(x)=\frac{1}{s^{\gamma}} p_{1}^{(\alpha)}\left(\frac{x}{s^{\gamma}}\right), \quad s>0
$$


we obtain

$$
\begin{aligned}
P\left(T_{\{1\}}\left(X_{\alpha}\right)<t\right) & =\frac{\Gamma(1-\gamma) p_{1}^{(\alpha)}(0)}{u_{1}^{(\alpha)}(0)} \int_{0}^{t} \frac{(t-s)^{\gamma-1} s^{-\gamma}}{\Gamma(\gamma) \Gamma(1-\gamma)} \cdot \frac{p_{1}^{(\alpha)}\left(1 / s^{\gamma}\right)}{p_{1}^{(\alpha)}(0)} \mathrm{d} s \\
& =\int_{0}^{1} \frac{(1-s)^{\gamma-1} s^{-\gamma}}{\Gamma(\gamma) \Gamma(1-\gamma)} \cdot \frac{p_{1}^{(\alpha)}\left(1 /(t s)^{\gamma}\right)}{p_{1}^{(\alpha)}(0)} \mathrm{d} s \\
& =P\left(\mathcal{R}_{\alpha}>\frac{1}{\left(t \mathcal{B}_{1-\gamma, \gamma}\right)^{\gamma}}\right) \quad(\text { from (2.76) }) \\
& =P\left(\frac{1}{\left(\mathcal{R}_{\alpha}\right)^{\alpha} \mathcal{B}_{1-\gamma, \gamma}}<t\right) .
\end{aligned}
$$

Now the proof is complete.

\subsection{Laplace transform formula for first hitting time of two points}

For later use, we prepare several important formulae concerning Laplace transforms for first hitting time of two points.

Let us denote the symmetric $\alpha$-stable process starting from $x \in \mathbb{R}$ by $X_{\alpha}^{x}(t)=x+$ $X_{\alpha}(t)$. Suppose that $1<\alpha \leq 2$. Recall that the Laplace transform of first hitting time of a single point is given by (see (5.101) )

$$
\varphi_{x \rightarrow a}^{q}:=E\left[\mathrm{e}^{-q T_{\{a\}}\left(X_{\alpha}^{x}\right)}\right]=\frac{u_{q}^{(\alpha)}(x-a)}{u_{q}^{(\alpha)}(0)} .
$$

The Laplace transform of first hitting time of two points, i.e., $T_{\{a\}}\left(X_{\alpha}^{x}\right) \wedge T_{\{b\}}\left(X_{\alpha}^{x}\right)$, is given by the following formula:

Proposition 5.4. Suppose that $1<\alpha \leq 2$. Let $x, a, b \in \mathbb{R}$. Then

$$
\varphi_{x \rightarrow a, b}^{q}:=E\left[\mathrm{e}^{-q T_{\{a\}}\left(X_{\alpha}^{x}\right) \wedge T_{\{b\}}\left(X_{\alpha}^{x}\right)}\right]=\frac{u_{q}^{(\alpha)}(x-a)+u_{q}^{(\alpha)}(x-b)}{u_{q}^{(\alpha)}(0)+u_{q}^{(\alpha)}(a-b)} .
$$

Proof. For any closed set $F$, we denote

$$
T_{F}^{x}=T_{F}\left(X_{\alpha}^{x}\right)=\inf \left\{t>0: X_{\alpha}^{x}(t) \in F\right\} .
$$

Following [2, p.49], we introduce the capacitary measure as

$$
\mu_{F}^{q}(A)=q \int E\left[\mathrm{e}^{-q T_{F}^{z}} ; X_{\alpha}^{z}\left(T_{F}^{z}\right) \in A\right] \mathrm{d} z, \quad A \in \mathcal{B}(\mathbb{R}) .
$$

Now we apply Theorem II.2.7 of [2] and obtain

$$
\int \mu_{F}^{q}(\mathrm{~d} y) \int_{A} u_{q}^{(\alpha)}(x-y) \mathrm{d} x=\int_{A} E\left[\mathrm{e}^{-q T_{F}^{x}}\right] \mathrm{d} x, \quad A \in \mathcal{B}(\mathbb{R}),
$$


where we have used the fact that the process considered is symmetric. This implies that

$$
E\left[\mathrm{e}^{-q T_{F}^{x}}\right]=\int u_{q}^{(\alpha)}(x-y) \mu_{F}^{q}(\mathrm{~d} y)
$$

By definition of $\mu_{F}^{q}$, we obtain

$$
E\left[\mathrm{e}^{-q T_{F}^{x}}\right]=q \int E\left[\mathrm{e}^{-q T_{F}^{z}} u_{q}^{(\alpha)}\left(x-X_{\alpha}^{z}\left(T_{F}^{z}\right)\right)\right] \mathrm{d} z .
$$

Now we let $F=\{a, b\}$. Then we have $T_{F}^{x}=T_{\{a\}}^{x} \wedge T_{\{b\}}^{x}$. Noting that $X_{\alpha}^{z}\left(T_{F}^{z}\right)=a$ or $b$ almost surely, we have

$$
E\left[\mathrm{e}^{-q T_{F}^{x}}\right]=C_{a \prec b}^{q} u_{q}^{(\alpha)}(x-a)+C_{b \prec a}^{q} u_{q}^{(\alpha)}(x-b)
$$

where

$$
C_{a \prec b}^{q}=q \int E\left[\mathrm{e}^{-q T_{F}^{z}} ; T_{\{a\}}^{z}<T_{\{b\}}^{z}\right] \mathrm{d} z .
$$

Since $T_{F}^{a}=T_{F}^{b}=0$ almost surely, we have

$$
\begin{aligned}
& 1=C_{a \prec b}^{q} u_{q}^{(\alpha)}(0)+C_{b \prec a}^{q} u_{q}^{(\alpha)}(a-b), \\
& 1=C_{a \prec b}^{q} u_{q}^{(\alpha)}(b-a)+C_{b \prec a}^{q} u_{q}^{(\alpha)}(0) .
\end{aligned}
$$

Hence we obtain

$$
C_{a \prec b}^{q}=C_{b \prec a}^{q}=\frac{1}{u_{q}^{(\alpha)}(0)+u_{q}^{(\alpha)}(a-b)} .
$$

Combining this with (5.32), we obtain the desired result.

The Laplace transform of first hitting time of point $a$ before hitting $b$ is given by the following formula:

Proposition 5.5. Suppose that $1<\alpha \leq 2$. Let $x, a, b \in \mathbb{R}$ with $a \neq b$. Then

$$
\begin{aligned}
\varphi_{x \rightarrow a \prec b}^{q} & :=E\left[\mathrm{e}^{-q T_{\{a\}}\left(X_{\alpha}^{x}\right)} ; T_{\{a\}}\left(X_{\alpha}^{x}\right)<T_{\{b\}}\left(X_{\alpha}^{x}\right)\right] \\
& =\frac{u_{q}^{(\alpha)}(0) u_{q}^{(\alpha)}(x-a)-u_{q}^{(\alpha)}(a-b) u_{q}^{(\alpha)}(x-b)}{\left\{u_{q}^{(\alpha)}(0)\right\}^{2}-\left\{u_{q}^{(\alpha)}(a-b)\right\}^{2}} .
\end{aligned}
$$

Proof. Let us keep the notations in the proof of Proposition 5.4. Noting that

$$
T_{\{a\}}^{x}=T_{\{b\}}^{x}+T_{\{a\}}^{x} \circ \theta_{T_{\{b\}}^{x}} \quad \text { on }\left\{T_{\{a\}}^{x}>T_{\{b\}}^{x}\right\},
$$

we see, by the strong Markov property, that

$$
E\left[\mathrm{e}^{-q T_{\{a\}}^{x}} ; T_{\{a\}}^{x}>T_{\{b\}}^{x}\right]=\varphi_{x \rightarrow b \prec a}^{q} \varphi_{b \rightarrow a}^{q} .
$$


Thus we have

$$
\varphi_{x \rightarrow a}^{q}=\varphi_{x \rightarrow a \prec b}^{q}+\varphi_{x \rightarrow b \prec a}^{q} \varphi_{b \rightarrow a}^{q} .
$$

Combining this with the trivial identity

$$
\varphi_{x \rightarrow a, b}^{q}=\varphi_{x \rightarrow a \prec b}^{q}+\varphi_{x \rightarrow b \prec a}^{q},
$$

we obtain

$$
\varphi_{x \rightarrow a \prec b}^{q}=\frac{\varphi_{x \rightarrow a}^{q}-\varphi_{b \rightarrow a}^{q} \varphi_{x \rightarrow a, b}^{q}}{1-\varphi_{b \rightarrow a}^{q}} .
$$

This proves the desired result.

Remark 5.6. The formula (5.38) can be written as

$$
\begin{aligned}
& E\left[\mathrm{e}^{-q T_{\{a\}}\left(X_{\alpha}^{x}\right)} ; T_{\{a\}}\left(X_{\alpha}^{x}\right)<T_{\{b\}}\left(X_{\alpha}^{x}\right)\right] \\
= & \frac{u_{q}^{(\alpha)}(x-b) h_{q}^{(\alpha)}(a-b)+u_{q}^{(\alpha)}(0)\left\{h_{q}^{(\alpha)}(x-b)-h_{q}^{(\alpha)}(x-a)\right\}}{\left\{u_{q}^{(\alpha)}(0)+u_{q}^{(\alpha)}(a-b)\right\} h_{q}^{(\alpha)}(a-b)} .
\end{aligned}
$$

Letting $q \rightarrow 0+$, we obtain

$$
P\left(T_{\{a\}}\left(X_{\alpha}^{x}\right)<T_{\{b\}}\left(X_{\alpha}^{x}\right)\right)=\frac{1}{2}\left\{1+\frac{|x-b|^{\alpha-1}-|x-a|^{\alpha-1}}{|a-b|^{\alpha-1}}\right\},
$$

which is a special case of Getoor's formula [16, Thm.6.5]. See also [48, Thm.6.1] for its application to Itô's measure for symmetric Lévy processes.

Remark 5.7. Let $a<x<b$. Then, as corollaries of Propositions 5.4 and 5.5, we recover the following well-known formulae (see, e.g., [24, Problem 1.7.6]) for the Brownian motion $\left(B^{x}(t)=x+B(t): t \geq 0\right)$ starting from $x$ :

$$
E\left[\mathrm{e}^{-q T_{\{a\}}\left(B^{x}\right) \wedge T_{\{b\}}\left(B^{x}\right)}\right]=\frac{\cosh \left(\sqrt{2 q}\left(x-\frac{b+a}{2}\right)\right)}{\cosh \left(\sqrt{2 q} \cdot \frac{b-a}{2}\right)}
$$

and

$$
E\left[\mathrm{e}^{-q T_{\{a\}}\left(B^{x}\right)} ; T_{\{a\}}\left(B^{x}\right)<T_{\{b\}}\left(B^{x}\right)\right]=\frac{\sinh \sqrt{2 q}(b-x)}{\sinh \sqrt{2 q}(b-a)} .
$$

\subsection{The Laplace transforms of $G_{\{a\}}\left(X_{\alpha}\right)$ and $\Xi_{\{a\}}\left(X_{\alpha}\right)$}

The following theorem generalises the formulae (5.6) and (5.7):

Theorem 5.8. Suppose that $1<\alpha \leq 2$. Let $a \neq 0$. Then it holds that

$$
E\left[\mathrm{e}^{-q G_{\{a\}}\left(X_{\alpha}\right)}\right]=\frac{\left\{u_{q}^{(\alpha)}(0)\right\}^{2}-\left\{u_{q}^{(\alpha)}(a)\right\}^{2}}{2 h^{(\alpha)}(a) u_{q}^{(\alpha)}(0)}
$$

and that

$$
E\left[\mathrm{e}^{-q \Xi_{\{a\}}\left(X_{\alpha}\right)}\right]=\frac{u_{q}^{(\alpha)}(a)}{u_{q}^{(\alpha)}(0)} \cdot \frac{2 h^{(\alpha)}(a) u_{q}^{(\alpha)}(0)}{\left\{u_{q}^{(\alpha)}(0)\right\}^{2}-\left\{u_{q}^{(\alpha)}(a)\right\}^{2}} .
$$


Remark 5.9. The left hand sides of (5.49) and (5.50) are functions of $q|a|^{\alpha}$ since

$$
G_{\{a\}}\left(X_{\alpha}\right) \stackrel{\text { law }}{=}|a|^{\alpha} G_{\{1\}}\left(X_{\alpha}\right) \quad \text { and } \quad \Xi_{\{a\}}\left(X_{\alpha}\right) \stackrel{\text { law }}{=}|a|^{\alpha} \Xi_{\{1\}}\left(X_{\alpha}\right) .
$$

We may check that so are the right hand sides by the following formulae:

$$
u_{q}^{(\alpha)}(0)=|a|^{\alpha-1} u_{q|a|^{\alpha}}^{(\alpha)}(0), u_{q}^{(\alpha)}(a)=|a|^{\alpha-1} u_{q|a|^{\alpha}}^{(\alpha)}(1) \text { and } h^{(\alpha)}(a)=|a|^{\alpha-1} h^{(\alpha)}(1) .
$$

For the proof of Theorem 5.8, we need the following proposition.

Proposition 5.10. Suppose that $X=X_{\alpha}$ with $1<\alpha \leq 2$. Let $a \neq 0$ and $q, r>0$. Then

$$
\boldsymbol{n}^{(\alpha)}\left[\mathrm{e}^{-q T_{\{a\}}-r\left(\zeta-T_{\{a\}}\right)} ; T_{\{a\}}<\zeta\right]=\frac{u_{r}^{(\alpha)}(a)}{u_{r}^{(\alpha)}(0)} \cdot \frac{u_{q}^{(\alpha)}(a)}{\left\{u_{q}^{(\alpha)}(0)\right\}^{2}-\left\{u_{q}^{(\alpha)}(a)\right\}^{2}} .
$$

Consequently, it holds that

$$
\boldsymbol{n}^{(\alpha)}\left[\mathrm{e}^{-q T_{\{a\}}} ; T_{\{a\}}<\zeta\right]=\frac{u_{q}^{(\alpha)}(a)}{\left\{u_{q}^{(\alpha)}(0)\right\}^{2}-\left\{u_{q}^{(\alpha)}(a)\right\}^{2}}
$$

and that

$$
\boldsymbol{n}^{(\alpha)}\left(T_{\{a\}}<\zeta\right)=\frac{1}{2 h^{(\alpha)}(a)} .
$$

Proof. Let us only prove the formula (5.53); in fact, from this formula we can obtain the formulae (5.54) and (5.55) immediately by the limit (4.6) and Lemma 4.1.

Let $\varepsilon>0$. By the strong Markov property of $\boldsymbol{n}^{(\alpha)}$, we have

$$
\begin{aligned}
& \boldsymbol{n}^{(\alpha)}\left[\mathrm{e}^{-q T_{\{a\}}-r\left(\zeta-T_{\{a\}}\right)} ; \varepsilon<T_{\{a\}}<\zeta\right] \\
= & \mathrm{e}^{-q \varepsilon} \boldsymbol{n}^{(\alpha)}\left[\left.\left(\varphi_{x \rightarrow a \prec 0}^{q}\right)\right|_{x=X(\varepsilon)} \varphi_{0 \rightarrow a}^{r} ; \varepsilon<T_{\{a\}} \wedge \zeta\right] \\
= & \mathrm{e}^{-q \varepsilon} \varphi_{0 \rightarrow a}^{r} E^{h^{(\alpha)}}\left[\left.\left(\frac{\varphi_{x \rightarrow a \prec 0}^{q}}{h^{(\alpha)}(x)}\right)\right|_{x=X(\varepsilon)} ; \varepsilon<T_{\{a\}}\right] .
\end{aligned}
$$

Here we used Theorem 4.2, Note that

$$
\begin{aligned}
& \frac{\varphi_{x \rightarrow a \prec 0}^{q}}{h^{(\alpha)}(x)} \cdot\left\{\left\{u_{q}^{(\alpha)}(0)\right\}^{2}-\left\{u_{q}^{(\alpha)}(a)\right\}^{2}\right\} \\
= & u_{q}^{(\alpha)}(a) \cdot \frac{h_{q}^{(\alpha)}(x)}{h^{(\alpha)}(x)}-u_{q}^{(\alpha)}(0) \cdot \frac{u_{q}^{(\alpha)}(x-a)-u_{q}^{(\alpha)}(a)}{h^{(\alpha)}(x)} .
\end{aligned}
$$

Suppose that $1<\alpha<2$. Then we see that the right hand side of (5.59) converges to $u_{q}^{(\alpha)}(a)$ as $x \rightarrow 0$ by Theorem 4.4. Letting $\varepsilon \rightarrow 0+$ in the identity (5.56)-(5.58), we obtain the formula (5.53) by the dominated convergence theorem.

Suppose that $\alpha=2$. We may assume without loss of generality that $a>0$. Then we see that the quantity (5.58) is equal to

$$
\frac{1}{2} \mathrm{e}^{-q \varepsilon} \varphi_{0 \rightarrow a}^{r} E^{h^{(2)}}\left[\left.\left(\frac{\varphi_{x \rightarrow a \prec 0}^{q}}{h^{(2)}(x)}\right)\right|_{x=X(\varepsilon)} ; \varepsilon<T_{\{a\}}, X(\varepsilon)>0\right] ;
$$


In fact, $P^{h^{(2)}}$ is nothing else but the law of the symmetrisation of the 3-dimensional Bessel process starting from the origin. We also see that the right hand side of (5.59) converges to $2 u_{q}^{(\alpha)}(a)$ as $x \rightarrow+0$ by Theorem 4.4. Hence, letting $\varepsilon \rightarrow 0+$ in the identity (5.56)(5.60), we obtain the formula (5.53) by the dominated convergence theorem. Now the proof is complete.

Now we proceed to prove Theorem 5.8 .

Proof of Theorem 5.8. Using the formulae (5.55) and (5.53) (with $r=q$ ), we have

$$
\begin{aligned}
& \boldsymbol{n}^{(\alpha)}\left[1-\mathrm{e}^{-q \zeta} ; T_{\{a\}}>\zeta\right] \\
= & \boldsymbol{n}^{(\alpha)}\left[1-\mathrm{e}^{-q \zeta}\right]-\boldsymbol{n}^{(\alpha)}\left(T_{\{a\}}<\zeta\right)+\boldsymbol{n}^{(\alpha)}\left[\mathrm{e}^{-q \zeta} ; T_{\{a\}}<\zeta\right] \\
= & \frac{1}{u_{q}^{(\alpha)}(0)}-\frac{1}{2 h^{(\alpha)}(a)}+\frac{u_{q}^{(\alpha)}(a)}{u_{q}^{(\alpha)}(0)} \cdot \frac{u_{q}^{(\alpha)}(a)}{\left\{u_{q}^{(\alpha)}(0)\right\}^{2}-\left\{u_{q}^{(\alpha)}(a)\right\}^{2}} .
\end{aligned}
$$

Hence we obtain

$$
\frac{\boldsymbol{n}^{(\alpha)}\left[1-\mathrm{e}^{-q \zeta} ; T_{\{a\}}>\zeta\right]}{\boldsymbol{n}^{(\alpha)}\left(T_{\{a\}}<\zeta\right)}=\frac{2 h^{(\alpha)}(a) u_{q}^{(\alpha)}(0)}{\left\{u_{q}^{(\alpha)}(0)\right\}^{2}-\left\{u_{q}^{(\alpha)}(a)\right\}^{2}}-1 .
$$

Combining this with the formula (3.17), we obtain (5.49).

Using the formulae (5.54) and (5.55), we have

$$
\frac{\boldsymbol{n}^{(\alpha)}\left[\mathrm{e}^{-q T_{\{a\}}} ; T_{\{a\}}<\zeta\right]}{\boldsymbol{n}^{(\alpha)}\left(T_{\{a\}}<\zeta\right)}=\frac{2 h^{(\alpha)}(a) u_{q}^{(\alpha)}(a)}{\left\{u_{q}^{(\alpha)}(0)\right\}^{2}-\left\{u_{q}^{(\alpha)}(a)\right\}^{2}} .
$$

Combining this with the formula (3.18), we obtain (5.50).

\subsection{Overshoots at the first passage time of a level}

For comparison with the description of the law of a first hitting time, we recall the law of the overshoot at the first passage time of a level. Let $X_{\alpha}=\left(X_{\alpha}(t): t \geq 0\right)$ denote the symmetric stable Lévy process of index $0<\alpha \leq 2$ starting from the origin such that $E\left[\mathrm{e}^{i \lambda X_{\alpha}(t)}\right]=\mathrm{e}^{-t|\lambda|^{\alpha}}$.

Let us consider the first passage time of level $a>0$ for $X_{\alpha}$ :

$$
T_{[a, \infty)}\left(X_{\alpha}\right)=\inf \left\{t>0: X_{\alpha}(t) \geq a\right\} .
$$

The variable $X_{\alpha}\left(T_{[a, \infty)}\left(X_{\alpha}\right)\right)-a$ is the overshoot at the first hitting time of level $a$.

The following theorem is due to Ray [34], although he does not express his result like this:

Theorem 5.11 ([34]). Suppose that $0<\alpha \leq 2$. Let $a>0$. Then

$$
X_{\alpha}\left(T_{[a, \infty)}\left(X_{\alpha}\right)\right)-a \stackrel{\text { law }}{=} a \frac{\mathcal{G}_{1-\frac{\alpha}{2}}}{\widehat{\mathcal{G}}_{\frac{\alpha}{2}}}
$$

where $\mathcal{G}_{1-\frac{\alpha}{2}}$ and $\widehat{\mathcal{G}}_{\frac{\alpha}{2}}$ are independent gamma variables of indices $1-\frac{\alpha}{2}$ and $\frac{\alpha}{2}$, respectively.

For its multidimensional analogue, see Blumenthal-Getoor-Ray [5]. 


\section{First hitting time of a single point for $\left|X_{\alpha}\right|$}

\subsection{The case of one-dimensional reflecting Brownian motion}

We consider the first hitting time of $a>0$ for the reflecting Brownian motion $|B|=$ $(|B|(t))$ :

$$
T_{\{a\}}(|B|)=\inf \{t>0:|B(t)|=a\}=T_{\{a\}}(B) \wedge T_{\{-a\}}(B) .
$$

It is well-known (see, e.g., [35, Prop.II.3.7]) that the law of the hitting time is of (SD) type where its Laplace transforms is given as follows:

$$
E\left[\mathrm{e}^{i \theta \widehat{B}\left(T_{\{a\}}(|B|)\right)}\right]=E\left[\mathrm{e}^{-\frac{1}{2} \theta^{2} T_{\{a\}}(|B|)}\right]=\frac{1}{\cosh (a \theta)}, \quad \theta \in \mathbb{R} .
$$

The identity (6.2) can be expressed as

$$
\widehat{B}\left(T_{\{a\}}(|B|)\right) \stackrel{\text { law }}{=} a \mathbb{C}_{1} \stackrel{\text { law }}{=} 2 a \mathcal{M}_{0}, \quad T_{\{a\}}(|B|) \stackrel{\text { law }}{=} a^{2} C_{1}
$$

Noting that

$$
\frac{1}{\cosh (a \theta)}=\frac{2 \mathrm{e}^{-a|\theta|}}{1+\mathrm{e}^{-2 a|\theta|}}=2 \mathrm{e}^{-a|\theta|} \sum_{n=0}^{\infty}(-1)^{n} \mathrm{e}^{-2 n a|\theta|},
$$

we have the following expansion:

$$
E\left[\mathrm{e}^{-q T_{\{a\}}(|B|)}\right]=2 \sum_{n=0}^{\infty}(-1)^{n} E\left[\mathrm{e}^{-q T_{\{(2 n+1) a\}}(B)}\right], \quad q>0 .
$$

Let us consider the random times $G_{\{a\}}(|B|)$ and $\Xi_{\{a\}}(|B|)$. By means of random time-change, Williams' path decomposition (Theorem 5.1) is also valid for the reflecting Brownian motion $|B|$ instead of $B$. Hence we may compute the Laplace transforms of these variables as follows:

$$
E\left[\mathrm{e}^{-q G_{\{a\}}(|B|)}\right]=\int_{0}^{a} \frac{\mathrm{d} m}{a}\left(E\left[\mathrm{e}^{-q T_{\{m\}}(|B|)}\right]\right)^{2}=\frac{\tanh (\sqrt{2 q} a)}{\sqrt{2 q} a}, \quad q>0
$$

and

$$
E\left[\mathrm{e}^{-q \Xi_{\{a\}}(|B|)}\right]=E\left[\mathrm{e}^{-q T_{\{a\}}(R)}\right]=\frac{\sqrt{2 q}|a|}{\sinh (\sqrt{2 q}|a|)}, \quad q>0 .
$$

In other words, we have

$$
G_{\{a\}}(|B|) \stackrel{\text { law }}{=} a^{2} T_{1}, \quad \Xi_{\{a\}}(|B|) \stackrel{\text { law }}{=} T_{\{a\}}(R) \stackrel{\text { law }}{=} a^{2} S_{1} .
$$




\subsection{Discussions about the Laplace transform of $T_{\{a\}}\left(\left|X_{\alpha}\right|\right)$}

Let us consider the first hitting time of point $a>0$ for $\left|X_{\alpha}\right|$ of index $1<\alpha \leq 2$ :

$$
T_{\{a\}}\left(\left|X_{\alpha}\right|\right)=\inf \left\{t>0:\left|X_{\alpha}(t)\right|=a\right\}=T_{\{a\}}\left(X_{\alpha}\right) \wedge T_{\{-a\}}\left(X_{\alpha}\right) .
$$

The following theorem generalises the Laplace transform formula (6.2) and the expansion (6.5).

Theorem 6.1. Suppose that $1<\alpha \leq 2$. Let $a \in \mathbb{R}$. Then

$$
\begin{aligned}
E\left[\mathrm{e}^{-q T_{\{a\}}\left(\left|X_{\alpha}\right|\right)}\right] & =\frac{2 u_{q}^{(\alpha)}(a)}{u_{q}^{(\alpha)}(0)+u_{q}^{(\alpha)}(2 a)} \\
& =2 \sum_{n=0}^{\infty}(-1)^{n} E\left[\mathrm{e}^{-q\left\{T_{\{a\}}\left(X_{\alpha}\right)+T_{\{2 a\}}\left(X_{\alpha}^{(1)}\right)+\cdots+T_{\{2 a\}}\left(X_{\alpha}^{(n)}\right)\right\}}\right]
\end{aligned}
$$

where $X_{\alpha}^{(1)}, \ldots, X_{\alpha}^{(n)}, \ldots$ are independent copies of $X_{\alpha}$.

Proof. Applying Proposition 5.4 with $x=0$ and $b=-a$, we obtain the first identity (6.10). Expanding the right hand side, we have

$$
E\left[\mathrm{e}^{-q T_{\{a\}}\left(\left|X_{\alpha}\right|\right)}\right]=\frac{2 u_{q}^{(\alpha)}(a)}{u_{q}^{(\alpha)}(0)} \sum_{n=0}^{\infty}(-1)^{n}\left\{\frac{u_{q}^{(\alpha)}(2 a)}{u_{q}^{(\alpha)}(0)}\right\}^{n} .
$$

Using the formula (5.25), we may rewrite the identity as

$$
E\left[\mathrm{e}^{-q T_{\{a\}}\left(\left|X_{\alpha}\right|\right)}\right]=2 E\left[\mathrm{e}^{-q T_{\{a\}}\left(X_{\alpha}\right)}\right] \sum_{n=0}^{\infty}(-1)^{n}\left\{E\left[\mathrm{e}^{-q T_{\{2 a\}}\left(X_{\alpha}\right)}\right]\right\}^{n} .
$$

This is nothing else but the second identity (6.11).

In the case of Brownian motion $B=(B(t))$ on one hand, we have

$$
T_{\{a\}}(B)+T_{\{2 a\}}\left(B^{(1)}\right)+\cdots+T_{\{2 a\}}\left(B^{(n)}\right) \stackrel{\text { law }}{=} T_{\{(2 n+1) a\}}(B)
$$

where $B^{(1)}, \ldots, B^{(n)}$ are independent copies of $B$. In the case of symmetric $\alpha$-stable process $X_{\alpha}=\left(X_{\alpha}(t)\right)$ for $1<\alpha<2$ on the other hand, however, the law of the sum

$$
T_{\{a\}}\left(X_{\alpha}\right)+T_{\{2 a\}}\left(X_{\alpha}^{(1)}\right)+\cdots+T_{\{2 a\}}\left(X_{\alpha}^{(n)}\right)
$$

differs from that of $T_{\{(2 n+1) a\}}\left(X_{\alpha}\right)$. In fact, we have the following theorem.

Theorem 6.2. Suppose that $1<\alpha \leq 2$. Let $a \in \mathbb{R}$. Then, for any $q>0$ and $n \geq 1$,

$$
E\left[\mathrm{e}^{-q\left\{T_{\{a\}}\left(X_{\alpha}\right)+T_{\{2 a\}}\left(X_{\alpha}^{(1)}\right)+\cdots+T_{\{2 a\}}\left(X_{\alpha}^{(n)}\right)\right\}}\right]<E\left[\mathrm{e}^{-q T_{\{(2 n+1) a\}}\left(X_{\alpha}\right)}\right] .
$$


Proof. Set

$$
D_{n}=E\left[\mathrm{e}^{-q T_{\{(2 n+1) a\}}\left(X_{\alpha}\right)}\right]-E\left[\mathrm{e}^{-q T_{\{(2 n-1) a\}}\left(X_{\alpha}\right)}\right] E\left[\mathrm{e}^{-q T_{\{2 a\}}\left(X_{\alpha}\right)}\right] .
$$

Then it suffices to prove that $D_{n}>0$ for all $n \geq 1$.

Let us keep the notations in the proof of Proposition 5.4. Note that

$$
D_{n}=\varphi_{0 \rightarrow(2 n+1) a}^{q}-\varphi_{0 \rightarrow(2 n-1) a}^{q} \varphi_{0 \rightarrow 2 a}^{q} .
$$

Using the formula (5.41) and the translation invariance, we have

$$
\begin{aligned}
\varphi_{0 \rightarrow(2 n+1) a}^{q} & =\varphi_{0 \rightarrow(2 n+1) a \prec(2 n-1) a}^{q}+\varphi_{0 \rightarrow(2 n-1) a \prec(2 n+1) a}^{q} \varphi_{(2 n-1) a \rightarrow(2 n+1) a}^{q} \\
& =\varphi_{0 \rightarrow(2 n+1) a \prec(2 n-1) a}^{q}+\varphi_{0 \rightarrow(2 n-1) a \prec(2 n+1) a}^{q} \varphi_{0 \rightarrow 2 a}^{q} .
\end{aligned}
$$

Using the formula (5.41), the translation invariance, and the symmetry, we have

$$
\varphi_{0 \rightarrow(2 n-1) a}^{q}=\varphi_{0 \rightarrow(2 n-1) a \prec(2 n+1) a}^{q}+\varphi_{0 \rightarrow(2 n+1) a \prec(2 n-1) a}^{q} \varphi_{0 \rightarrow 2 a}^{q} .
$$

Hence we obtain

$$
D_{n}=\varphi_{0 \rightarrow(2 n+1) a \prec(2 n-1) a}^{q}\left\{1-\left(\varphi_{0 \rightarrow 2 a}^{q}\right)^{2}\right\},
$$

which turns out to be positive because both $\varphi_{0 \rightarrow(2 n+1) a \prec(2 n-1) a}^{q}$ and $\varphi_{0 \rightarrow 2 a}^{q}$ are positive and less than 1 . Now the proof is complete.

Remark 6.3. The consistency of the two formulae (6.18) and (6.22) can be confirmed by the formulae (5.25) and (5.38) as follows:

$$
\begin{aligned}
& \varphi_{0 \rightarrow(2 n+1) a \prec(2 n-1) a}^{q}\left\{1-\left(\varphi_{0 \rightarrow 2 a}^{q}\right)^{2}\right\} \\
= & \frac{u_{q}^{(\alpha)}(0) u_{q}^{(\alpha)}((2 n+1) a)-u_{q}^{(\alpha)}(2 a) u_{q}^{(\alpha)}((2 n-1) a)}{\left\{u_{q}^{(\alpha)}(0)\right\}^{2}-\left\{u_{q}^{(\alpha)}(2 a)\right\}^{2}} \cdot\left\{1-\left(\frac{u_{q}^{(\alpha)}(2 a)}{u_{q}^{(\alpha)}(0)}\right)^{2}\right\} \\
= & \frac{u_{q}^{(\alpha)}((2 n+1) a)}{u_{q}^{(\alpha)}(0)}-\frac{u_{q}^{(\alpha)}((2 n-1) a)}{u_{q}^{(\alpha)}(0)} \cdot \frac{u_{q}^{(\alpha)}(2 a)}{u_{q}^{(\alpha)}(0)} \\
= & \varphi_{0 \rightarrow(2 n+1) a}^{q}-\varphi_{0 \rightarrow(2 n-1) a}^{q} \varphi_{0 \rightarrow 2 a}^{q} .
\end{aligned}
$$

\subsection{The Laplace transforms of $G_{\{a\}}\left(\left|X_{\alpha}\right|\right)$ and $\Xi_{\{a\}}\left(\left|X_{\alpha}\right|\right)$}

Since $\left|X_{\alpha}\right|=\left(\left|X_{\alpha}(t)\right|: t \geq 0\right)$ is a strong Markov process, the arguments of Section 3.2 are valid for $X=\left|X_{\alpha}\right|$. Let us compute the Laplace transforms of $G_{\{a\}}\left(\left|X_{\alpha}\right|\right)$ and $\Xi_{\{a\}}\left(\left|X_{\alpha}\right|\right)$.

Theorem 6.4. Suppose that $1<\alpha \leq 2$. Let $a>0$. Then it holds that

$$
E\left[\mathrm{e}^{-q G_{\{a\}}\left(\left|X_{\alpha}\right|\right)}\right]=\frac{2 V_{q}^{(\alpha)}(a)}{\left\{u_{q}^{(\alpha)}(0)+u_{q}^{(\alpha)}(2 a)\right\}\left\{4 h^{(\alpha)}(a)-h^{(\alpha)}(2 a)\right\}}
$$


and that

$$
E\left[\mathrm{e}^{-q \Xi_{\{a\}}\left(\left|X_{\alpha}\right|\right)}\right]=\frac{u_{q}^{(\alpha)}(a)\left\{4 h^{(\alpha)}(a)-h^{(\alpha)}(2 a)\right\}}{V_{q}^{(\alpha)}(a)}
$$

where

$$
V_{q}^{(\alpha)}(a):=\left\{u_{q}^{(\alpha)}(0)\right\}^{2}+u_{q}^{(\alpha)}(0) u_{q}^{(\alpha)}(2 a)-2\left\{u_{q}^{(\alpha)}(a)\right\}^{2} .
$$

For the proof of Theorem 6.4, we need a certain Laplace transform formula for first hitting time of three points. Avoiding unnecessary generality, we are satisfied with the following special case:

Proposition 6.5. Suppose that $1<\alpha \leq 2$. Let $x, a \in \mathbb{R}$. Then

$$
\begin{aligned}
\varphi_{x \rightarrow 0, a,-a}^{q} & :=E\left[\mathrm{e}^{-q T_{\{0, a,-a\}}\left(X_{\alpha}^{x}\right)}\right] \\
& =C_{0 \prec a,-a}^{q} u_{q}(x)+C_{a \prec 0,-a}^{q} u_{q}(x-a)+C_{-a \prec 0, a}^{q} u_{q}(x+a)
\end{aligned}
$$

where

$$
C_{0 \prec a,-a}^{q}=\frac{u_{q}^{(\alpha)}(0)+u_{q}^{(\alpha)}(2 a)-2 u_{q}^{(\alpha)}(a)}{V_{q}^{(\alpha)}(a)}
$$

and

$$
C_{a \prec 0,-a}^{q}=C_{-a \prec 0, a}^{q}=\frac{u_{q}^{(\alpha)}(0)-u_{q}^{(\alpha)}(a)}{V_{q}^{(\alpha)}(a)} .
$$

The proof of Proposition 6.5 is similar to that of Proposition 5.4 based on the identity (5.31) with $F=\{0, a,-a\}$, so we omit it.

Proposition 6.6. Suppose that $1<\alpha \leq 2$. Let $x, a \in \mathbb{R}$ with $a \neq 0$. Then

$$
\begin{aligned}
\varphi_{x \rightarrow a,-a \prec 0}^{q} & :=E\left[\mathrm{e}^{-q T_{\{a,-a\}}\left(X_{\alpha}^{x}\right)} ; T_{\{a,-a\}}\left(X_{\alpha}^{x}\right)<T_{\{0\}}\left(X_{\alpha}^{x}\right)\right] \\
& =\frac{\varphi_{x \rightarrow a,-a}^{q}-\varphi_{0 \rightarrow a,-a}^{q} \varphi_{x \rightarrow 0, a,-a}^{q}}{1-\varphi_{0 \rightarrow a,-a}^{q}} \\
& =\frac{u_{q}^{(\alpha)}(0)\left\{u_{q}^{(\alpha)}(x-a)+u_{q}^{(\alpha)}(x+a)\right\}-2 u_{q}^{(\alpha)}(a) u_{q}^{(\alpha)}(x)}{V_{q}^{(\alpha)}(a)} .
\end{aligned}
$$

The proof of Proposition 6.6 is similar to that of Proposition 5.5, so we omit it.

Let $\boldsymbol{m}^{(\alpha)}$ denote Itô's measure for $\left|X_{\alpha}\right|$ corresponding to the local time satisfying (4.10). The following proposition is crucial to the proof of Theorem 6.4.

Proposition 6.7. Suppose that $1<\alpha \leq 2$. Let $a>0$ and $q, r>0$. Then

$$
\boldsymbol{m}^{(\alpha)}\left[\mathrm{e}^{-q T_{\{a\}}-r\left(\zeta-T_{\{a\}}\right)} ; T_{\{a\}}<\zeta\right]=\frac{u_{r}^{(\alpha)}(a)}{u_{r}^{(\alpha)}(0)} \cdot \frac{2 u_{q}^{(\alpha)}(a)}{V_{q}^{(\alpha)}(a)} .
$$


Consequently, it holds that

$$
\boldsymbol{m}^{(\alpha)}\left[\mathrm{e}^{-q T_{\{a\}}} ; T_{\{a\}}<\zeta\right]=\frac{2 u_{q}^{(\alpha)}(a)}{V_{q}^{(\alpha)}(a)}
$$

and that

$$
\boldsymbol{m}^{(\alpha)}\left(T_{\{a\}}<\zeta\right)=\frac{2}{4 h^{(\alpha)}(a)-h^{(\alpha)}(2 a)} .
$$

Proof of Proposition 6.7. By definitions of $\boldsymbol{n}^{(\alpha)}$ and $\boldsymbol{m}^{(\alpha)}$, we have

$$
\boldsymbol{m}^{(\alpha)}\left[\mathrm{e}^{-q T_{\{a\}}-r\left(\zeta-T_{\{a\}}\right)} ; T_{\{a\}}<\zeta\right]=\boldsymbol{n}^{(\alpha)}\left[\mathrm{e}^{-q T_{\{a,-a\}}-r\left(\zeta-T_{\{a,-a\}}\right)} ; T_{\{a,-a\}}<\zeta\right] .
$$

Let $\varepsilon>0$. Then we have

$$
\begin{aligned}
& \boldsymbol{n}^{(\alpha)}\left[\mathrm{e}^{-q T_{\{a,-a\}}-r\left(\zeta-T_{\{a,-a\}}\right)} ; \varepsilon<T_{\{a,-a\}}<\zeta\right] \\
= & \mathrm{e}^{-q \varepsilon} \boldsymbol{n}^{(\alpha)}\left[\left.\left(\varphi_{x \rightarrow a,-a \prec 0}^{q}\right)\right|_{x=X(\varepsilon)} \cdot \varphi_{a \rightarrow 0}^{r} ; \varepsilon<T_{\{a,-a\}} \wedge \zeta\right] \\
= & \mathrm{e}^{-q \varepsilon} \varphi_{a \rightarrow 0}^{r} E^{h^{(\alpha)}}\left[\left.\left(\frac{\varphi_{x \rightarrow a,-a \prec 0}^{q}}{h^{(\alpha)}(x)}\right)\right|_{x=X(\varepsilon)} ; \varepsilon<T_{\{a,-a\}}\right] .
\end{aligned}
$$

Here we utilised Theorem 4.2, Noting that, by Theorem 4.4, we have

$$
\lim _{x \rightarrow 0} \frac{u_{q}^{(\alpha)}(a-x)+u_{q}^{(\alpha)}(a+x)-2 u_{q}^{(\alpha)}(a)}{h^{(\alpha)}(x)}=0
$$

in whichever case where $1<\alpha<2$ or $\alpha=2$. Hence, we utilise Proposition 6.6 and obtain

$$
\lim _{x \rightarrow 0} \frac{\varphi_{x \rightarrow a,-a \prec 0}^{q}}{h^{(\alpha)}(x)}=\frac{2 u_{q}^{(\alpha)}(a)}{V_{q}^{(\alpha)}(a)} .
$$

Thus, letting $\varepsilon \rightarrow 0+$ in the formula (6.43), we obtain (6.37) by the dominated convergence theorem. By letting $r \rightarrow 0+$ in the formula (6.37), we obtain (6.38). Noting that

$$
V_{q}^{(\alpha)}(a)=2\left\{u_{q}^{(\alpha)}(0)+u_{q}^{(\alpha)}(a)\right\} h_{q}^{(\alpha)}(a)-u_{q}^{(\alpha)}(0) h_{q}^{(\alpha)}(2 a)
$$

we have

$$
\lim _{q \rightarrow 0+} \frac{2 u_{q}^{(\alpha)}(a)}{V_{q}^{(\alpha)}(a)}=\frac{2}{4 h^{(\alpha)}(a)-h^{(\alpha)}(2 a)} .
$$

Hence, by letting $q \rightarrow 0+$ in the formula (6.38), we obtain (6.39). Now the proof is complete.

The proof of Theorem 6.4 is now completely parallel to that of Theorem 5.8. Thus we omit the proof of Theorem 6.4. 


\section{Appendix: Computation of the constant $h^{(\alpha)}(1)$}

Proposition 7.1. For $1<\alpha<3$, it holds that

$$
\frac{1}{\pi} \int_{0}^{\infty} \frac{1-\cos x}{x^{\alpha}} \mathrm{d} x=\frac{1}{2 \Gamma(\alpha) \sin \frac{\pi(\alpha-1)}{2}} .
$$

As a check, the formula (7.1) in the case when $\alpha=2$ is equivalent via integration by parts to the well-known formula:

$$
\int_{0}^{\infty} \frac{\sin x}{x} \mathrm{~d} x=\frac{\pi}{2}
$$

Proof. We start with the identity:

$$
\int_{0}^{\infty} x^{\gamma-1} \mathrm{e}^{-z x} \mathrm{~d} x=\Gamma(\gamma) z^{-\gamma}
$$

for $\gamma>0$ and Re $z>0$. For $0<\alpha<1, \varepsilon>0$ and $\lambda \in \mathbb{R}$, we set $\gamma=1-\alpha$ and $z=\varepsilon-i \lambda$. Then we obtain

$$
\int_{0}^{\infty} \frac{\mathrm{e}^{i \lambda x} \mathrm{e}^{-\varepsilon x}}{x^{\alpha}} \mathrm{d} x=\Gamma(1-\alpha)(\varepsilon-i \lambda)^{\alpha-1} .
$$

Using the identity $\Gamma(2-\alpha)=(1-\alpha) \Gamma(1-\alpha)$ and subtracting (17.4) for $\lambda=0$ from that for $\lambda=\lambda$, we obtain

$$
\int_{0}^{\infty} \frac{\left(1-\mathrm{e}^{i \lambda x}\right) \mathrm{e}^{-\varepsilon x}}{x^{\alpha}} \mathrm{d} x=\Gamma(2-\alpha) \cdot \frac{\varepsilon^{\alpha-1}-(\varepsilon-i \lambda)^{\alpha-1}}{1-\alpha} .
$$

Rewriting the right hand side, we obtain

$$
\int_{0}^{\infty} \frac{\left(1-\mathrm{e}^{i \lambda x}\right) \mathrm{e}^{-\varepsilon x}}{x^{\alpha}} \mathrm{d} x=\Gamma(2-\alpha) \int_{\varepsilon}^{\varepsilon-i \lambda} z^{\alpha-2} \mathrm{~d} z
$$

where the integration on the right hand side is taken over a segment from $\{\varepsilon-i l: l \in \mathbb{R}\}$. Since both sides of (7.6) are analytic on $0<\operatorname{Re} \alpha<2$, we see, by analytic continuation, that the identity (7.6) remains true for $0<\alpha<2$.

Let us restrict ourselves to the case when $1<\alpha<2$. Taking the limit $\varepsilon \rightarrow 0+$ on both sides of the identity (7.6), we obtain

$$
\begin{aligned}
\int_{0}^{\infty} \frac{1-\mathrm{e}^{i \lambda x}}{x^{\alpha}} \mathrm{d} x & =\Gamma(2-\alpha) \int_{0}^{-i \lambda} z^{\alpha-2} \mathrm{~d} z \\
& =\Gamma(2-\alpha) \cdot \frac{(-i \lambda)^{\alpha-1}}{\alpha-1}
\end{aligned}
$$

where the branch of $f(w)=w^{\alpha-1}$ is chosen so that $f(1)=1$. Hence we obtain

$$
\int_{0}^{\infty} \frac{1-\mathrm{e}^{i \lambda x}}{x^{\alpha}} \mathrm{d} x=\Gamma(2-\alpha) \cdot \frac{\lambda^{\alpha-1}}{\alpha-1} \mathrm{e}^{-\frac{\pi(\alpha-1) i}{2}} .
$$


Taking the real parts on both sides, we obtain

$$
\int_{0}^{\infty} \frac{1-\cos \lambda x}{x^{\alpha}} \mathrm{d} x=\Gamma(2-\alpha) \cdot \frac{\lambda^{\alpha-1}}{\alpha-1} \cos \frac{\pi(\alpha-1)}{2} .
$$

Letting $\lambda=1$, we obtain

$$
\frac{1}{\pi} \int_{0}^{\infty} \frac{1-\cos x}{x^{\alpha}} \mathrm{d} x=\frac{\Gamma(2-\alpha)}{\pi(\alpha-1)} \cdot \cos \frac{\pi(\alpha-1)}{2} .
$$

(We may find the formula (7.11) also in [21, pp.88].) By a simple computation, we have

$$
\begin{aligned}
(\mathrm{RHS} \text { of (7.11) }) & =\frac{\Gamma(2-\alpha)}{\pi(\alpha-1)} \cdot \frac{\sin \pi(\alpha-1)}{2 \sin \frac{\pi(\alpha-1)}{2}} \\
& =\frac{1}{(\alpha-1) \Gamma(\alpha-1)} \cdot \frac{1}{2 \sin \frac{\pi(\alpha-1)}{2}} \\
& =\frac{1}{2 \Gamma(\alpha) \sin \frac{\pi(\alpha-1)}{2}} .
\end{aligned}
$$

Hence we have proved the identity (17.1) when $1<\alpha<2$. By analytic continuation, the identity (17.1) is proved to be valid also when $2 \leq \alpha<3$. Therefore the proof is complete.

\section{References}

[1] O. Barndorff-Nielsen, J. Kent, and M. Sørensen. Normal variance-mean mixtures and $z$ distributions. Internat. Statist. Rev., 50(2):145-159, 1982.

[2] J. Bertoin. Lévy processes, volume 121 of Cambridge Tracts in Mathematics. Cambridge University Press, Cambridge, 1996.

[3] J. Bertoin, T. Fujita, B. Roynette, and M. Yor. On a particular class of selfdecomposable random variables: the durations of Bessel excursions straddling independent exponential times. Probab. Math. Statist., 26(2):315-366, 2006.

[4] R. M. Blumenthal and R. K. Getoor. Markov processes and potential theory. Pure and Applied Mathematics, Vol. 29. Academic Press, New York, 1968.

[5] R. M. Blumenthal, R. K. Getoor, and D. B. Ray. On the distribution of first hits for the symmetric stable processes. Trans. Amer. Math. Soc., 99:540-554, 1961.

[6] L. Bondesson. On the infinite divisibility of the half-Cauchy and other decreasing densities and probability functions on the nonnegative line. Scand. Actuar. J., (34):225-247, 1987.

[7] L. Bondesson. Generalized gamma convolutions and related classes of distributions and densities, volume 76 of Lecture Notes in Statistics. Springer-Verlag, New York, 1992. 
[8] P. Bourgade, T. Fujita, and M. Yor. Euler's formulae for $\zeta(2 n)$ and products of Cauchy variables. Electron. Comm. Probab., 12:73-80 (electronic), 2007.

[9] J. Bretagnolle. Résultats de Kesten sur les processus à accroissements indépendants. In Séminaire de Probabilités, V (Univ. Strasbourg, année universitaire 1969-1970), pages 21-36. Lecture Notes in Math., Vol. 191. Springer, Berlin, 1971.

[10] P. Carmona, F. Petit, and M. Yor. On the distribution and asymptotic results for exponential functionals of Lévy processes. In Exponential functionals and principal values related to Brownian motion, Bibl. Rev. Mat. Iberoamericana, pages 73-130. Rev. Mat. Iberoamericana, Madrid, 1997.

[11] L. Chaumont and M. Yor. Exercises in probability, A guided tour from measure theory to random processes, via conditioning, volume 13 of Cambridge Series in Statistical and Probabilistic Mathematics. Cambridge University Press, Cambridge, 2003.

[12] L. Devroye. A note on Linnik's distribution. Statist. Probab. Lett., 9(4):305-306, 1990.

[13] C. Donati-Martin, B. Roynette, P. Vallois, and M. Yor. On constants related to the choice of the local time at 0 , and the corresponding Itô measure for Bessel processes with dimension $d=2(1-\alpha), 0<\alpha<1$. Studia Sci. Math. Hungar., 45(2):207-221, 2008.

[14] W. Feller. An introduction to probability theory and its applications. Vol. II. Second edition. John Wiley \& Sons Inc., New York, 1971.

[15] T. Fujita, Y. Yano, and M. Yor. in preparation.

[16] R. K. Getoor. Continuous additive functionals of a Markov process with applications to processes with independent increments. J. Math. Anal. Appl., 13:132-153, 1966.

[17] B. Grigelionis. Processes of Meixner type. Liet. Mat. Rink., 39(1):40-51, 1999.

[18] B. Grigelionis. Generalized z-distributions and related stochastic processes. Liet. Mat. Rink., 41(3):303-319, 2001.

[19] B. Grigelionis. On the self-decomposability of Euler's gamma function. Liet. Mat. Rink., 43(3):359-370, 2003.

[20] M. Hayashi and K. Yano. On the laws of total local times for h-paths of stable Lévy processes. in preparation.

[21] I. A. Ibragimov and Yu. V. Linnik. Independent and stationary sequences of random variables. Wolters-Noordhoff Publishing, Groningen, 1971. With a supplementary chapter by I. A. Ibragimov and V. V. Petrov, Translation from the Russian edited by J. F. C. Kingman. 
[22] N. Ikeda and S. Watanabe. Stochastic differential equations and diffusion processes, volume 24 of North-Holland Mathematical Library. North-Holland Publishing Co., Amsterdam, second edition, 1989.

[23] K. Itô. Poisson point processes attached to Markov processes. In Proceedings of the Sixth Berkeley Symposium on Mathematical Statistics and Probability (Univ. California, Berkeley, Calif., 1970/1971), Vol. III: Probability theory, pages 225-239, Berkeley, Calif., 1972. Univ. California Press.

[24] K. Itô and H. P. McKean, Jr. Diffusion processes and their sample paths. SpringerVerlag, Berlin, 1974. Second printing, corrected, Die Grundlehren der mathematischen Wissenschaften, Band 125.

[25] L. F. James. Gamma tilting calculus for GGC and Dirichlet means with applications to Linnik processes and occupation time laws for randomly skewed Bessel processes and bridges. preprint, arXiv:math/0610218, 2006.

[26] L. F. James, B. Roynette, and M. Yor. Generalized Gamma convolutions, Dirichlet means, Thorin measures with explicit examples. Probab. Surv., 5:346-415, 2008.

[27] H. Kesten. Hitting probabilities of single points for processes with stationary independent increments. Memoirs of the American Mathematical Society, No. 93. American Mathematical Society, Providence, R.I., 1969.

[28] F. B. Knight. Brownian local times and taboo processes. Trans. Amer. Math. Soc., 143:173-185, 1969.

[29] M. B. Marcus and J. Rosen. Markov processes, Gaussian processes, and local times, volume 100 of Cambridge Studies in Advanced Mathematics. Cambridge University Press, Cambridge, 2006.

[30] P. A. Meyer. Processus de Poisson ponctuels, d'après K. Ito. In Séminaire de Probabilités, V (Univ. Strasbourg, année universitaire 1969-1970), pages 177-190. Lecture Notes in Math., Vol. 191. Springer, Berlin, 1971.

[31] J. Pitman and M. Yor. Bessel processes and infinitely divisible laws. In Stochastic integrals (Proc. Sympos., Univ. Durham, Durham, 1980), volume 851 of Lecture Notes in Math., pages 285-370. Springer, Berlin, 1981.

[32] J. Pitman and M. Yor. Infinitely divisible laws associated with hyperbolic functions. Canad. J. Math., 55(2):292-330, 2003.

[33] J. Pitman and M. Yor. Itô's excursion theory and its applications. Jpn. J. Math., 2(1):83-96, 2007.

[34] D. Ray. Stable processes with an absorbing barrier. Trans. Amer. Math. Soc., 89:1624, 1958. 
[35] D. Revuz and M. Yor. Continuous martingales and Brownian motion, volume 293 of Grundlehren der Mathematischen Wissenschaften. Springer-Verlag, Berlin, third edition, 1999.

[36] B. Roynette, P. Vallois, and M. Yor. A family of generalized gamma convoluted variables. to appear in Prob. Math. Stat., 2009.

[37] P. Salminen. On last exit decompositions of linear diffusions. Studia Sci. Math. Hungar., 33(1-3):251-262, 1997.

[38] W. Schoutens. Stochastic processes and orthogonal polynomials, volume 146 of Lecture Notes in Statistics. Springer-Verlag, New York, 2000.

[39] W. Schoutens. Lévy processes in finance: Pricing financial derivatives. John Wiley \& Sons Inc., 2003.

[40] W. Schoutens and J. L. Teugels. Lévy processes, polynomials and martingales. Comm. Statist. Stochastic Models, 14(1-2):335-349, 1998. Special issue in honor of Marcel F. Neuts.

[41] D. N. Shanbhag and M. Sreehari. On certain self-decomposable distributions. $Z$. Wahrscheinlichkeitstheorie und Verw. Gebiete, 38(3):217-222, 1977.

[42] O. Thorin. On the infinite divisibility of the Pareto distribution. Scand. Actuar. J., (1):31-40, 1977.

[43] D. Williams. Decomposing the Brownian path. Bull. Amer. Math. Soc., 76:871-873, 1970.

[44] D. Williams. Path decomposition and continuity of local time for one-dimensional diffusions. I. Proc. London Math. Soc. (3), 28:738-768, 1974.

[45] M. Winkel. Electronic foreign-exchange markets and passage events of independent subordinators. J. Appl. Probab., 42(1):138-152, 2005.

[46] M. Yamazato. Topics related to gamma processes. In Stochastic processes and applications to mathematical finance, pages 157-182. World Sci. Publ., Hackensack, NJ, 2006.

[47] K. Yano. Convergence of excursion point processes and its applications to functional limit theorems of markov processes on a half line. Bernoulli, 14(4):963-987, 2008.

[48] K. Yano. Excursions away from a regular point for one-dimensional symmetric Lévy processes without Gaussian part. submitted. preprint, arXiv:0805.3881, 2008.

[49] K. Yano, Y. Yano, and M. Yor. Penalising symmetric stable Lévy paths. submitted. preprint, arXiv:0807.4336, 2008. 